\title{
Development of Robust Flight Control Laws for a Highly Flexible Aircraft in the Frequency Domain
}

\author{
Alexander Köthe* and Robert Luckner ${ }^{\dagger}$ \\ Technische Universität Berlin, Marchstrasse 12, 10587 Berlin, Germany \\ Pedro J. G. Ramirez ${ }^{\ddagger}$ and Flávio J. Silvestre ${ }^{\S}$ \\ Instituto Tecnológico de Aeronáutica, São José dos Campos, SP, 12228-900, Brazil \\ Zi Yang Pang ^ and Carlos E. S. Cesnik $\|$ \\ University of Michigan, Ann Arbor, MI, 48109-2410, USA
}

\begin{abstract}
Modern manned and unmanned aircraft designs have a lightweight and flexible structure to increase the flight performance. This trend is continuing with more flexible aircraft structures that possess a nonlinear behaviour. Typically those very flexible are equiped with a flight control system. This paper addresses trajectory control in longitudinal and lateral motion of a highly flexible aircraft with nonlinear dynamics. A new flight control design approach is introduced that is based on the classical multi-loop control law structure with specific considerations for the nonlinear structural behaviour. The inner loops of the flight control laws shall ensure stability and an optimal shape of the aircraft. The nonlinearities are summed up as uncertainties. Control design strategies in the frequency domain are used to achieve the design objectives. The outer loops are based on the classical multi-loop concept for autopilots. The design method and results in a linear and nonlinear simulation of the very flexible unmanned aerial vehicle X-HALE are presented.
\end{abstract}

\section{Nomenclature}

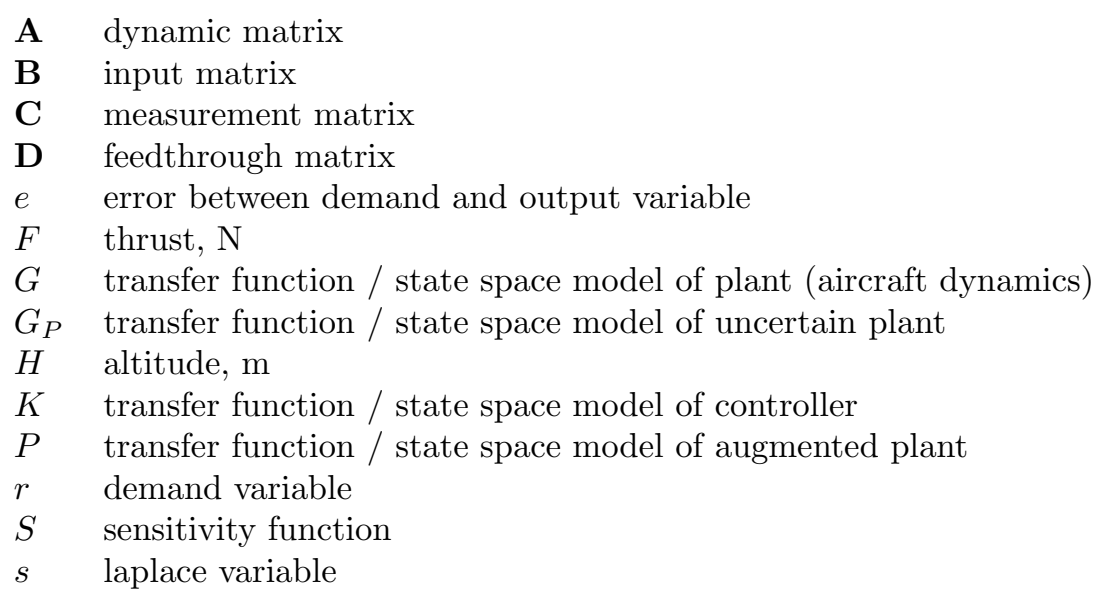

\footnotetext{
* Research Assistant and Ph.D. candidate, Department of Flight Mechanics, Flight Control and Aeroelasticity, Alexander.Koethe@ilr.tu-berlin.de

${ }^{\dagger}$ Full Professor, Department of Flight Mechanics, Flight Control and Aeroelasticity, Robert.Luckner@tu-berlin.de, Associate Fellow AIAA

¥Ph.D. candidate, Divisão de Engenharia Aeronáutica, Instituto Tecnológico de Aeronáutica, pedrojgonzalezr@gmail.com, AIAA Member

§Professor, Divisão de Engenharia Aeronáutica, Instituto Tecnológico de Aeronáutica, flaviojs@ita.br, AIAA Member

\PhD Candidate, Department of Aerospace Engineering, MI, pziyang@U-M.edu, Member AIAA

" Professor, Department of Aerospace Engineering, MI, cesnik@U-M.edu, Fellow AIAA
} 
complementary sensitivity function

time, $\mathrm{s}$

input variable

$V \quad$ velocity, $\mathrm{m} \mathrm{s}^{-1}$

$\mathbf{W}_{A}$ uncertainty weight matrix

$\mathbf{W}_{S}$ sensitivity weight matrix

$\mathbf{W}_{T}$ complementary sensitivity weight matrix

$\mathbf{W}_{U}$ input vector weight matrix

$w_{w}$ wind velocity in $z$ direction, $\mathrm{m} \mathrm{s}^{-1}$

w exogenuous input of augmented plant

$\mathrm{x}$ state variable

y output/ measurment variable

$\mathbf{z}$ exogenuous output of augmented plant

$\beta \quad$ sideslip angle, radian

$\varepsilon \quad$ curvature

$\eta \quad$ elevator deflection, radian

$\Theta$ pitch angle, radian

$\sigma \quad$ singular values

$\tau \quad$ time delay, $1 \mathrm{~s}$

$\xi \quad$ aileron deflection, radian

$\Phi$ bank angle, radian

$\Psi \quad$ azimuth angle, radian

$\omega_{B} \quad$ bandwidth, radian $\mathrm{s}^{-1}$

$\omega_{C} \quad$ cut off frequency, radian $\mathrm{s}^{-1}$

\section{Introduction}

Driven by increasing aircraft performance and simultaneous fuel reduction, the aircraft design has changed from rigid body structures to flexible, lightweight structures in the recent years. A high-aspect ratio wing reduces induced drag and improves mission capabilities such as heavier payload and greater range. This development can be seen in modern commercial aircraft like Boeing 787 or Airbus A 350 as well as in unmanned aerial vehicles such as High Altitude Long Endurance (HALE) aircraft. As wings become more and more flexible, higher deformations occur and the aeroelastic behaviour becomes nonlinear. As a consequence traditional linear design and simulation methods are no longer adequate. All of the previous mentioned aircraft have an electronic flight control system that has to ensure stability and performance even if the aircraft behaviour is nonlinear. In literature, flight control law design for such flexible aircraft is moving from traditional approaches to modern control theory techniques such as dynamic inversion, Linear Quadratic Regulator (LQR) and Linear Quadratic Gaussian (LQG) observers or robust control design. ${ }^{3,5-7,12}$ Those modern techniques are used for inner and outer loops. They consider the aircraft as acoupled multiple input, multiple output system of flight dynamic and aeroelastic states. Those approaches are successful, but the flight control laws are more complex and more difficult to understand compared to traditional flight control law design techniques. This paper presents a new design approach that uses (robust) frequency design techniques for the inner loop to damp structural and rigid body motions with a high bandwidth. The inner loop control laws ensure that the aircraft dynamic behaviour is similar to traditional rigid body aircraft. As a consequence, traditional outer loops for trajectory control in longitudinal and lateral motion can be used. The proof and validation of this concept is carried out with the highly flexible unmanned aerial vehicle X-HALE that was developed by the the University of Michigan (U-M). ${ }^{2}$

\section{Reference aircraft - Very Flexible UAV X-HALE}

The Instituto Tecnológico de Aeronáutica (ITA), the University of Michigan (U-M) and the Technische Universitt Berlin (TUB) cooperate in the investigation of different concepts for HALE aircraft. ITA and U-M study highly flexible aircraft structure while TUB is investigating multi-body-aircraft as alternative to flexible structures. ${ }^{9}$ New concepts for modelling and control of such an aircraft are investigated and demonstrated 


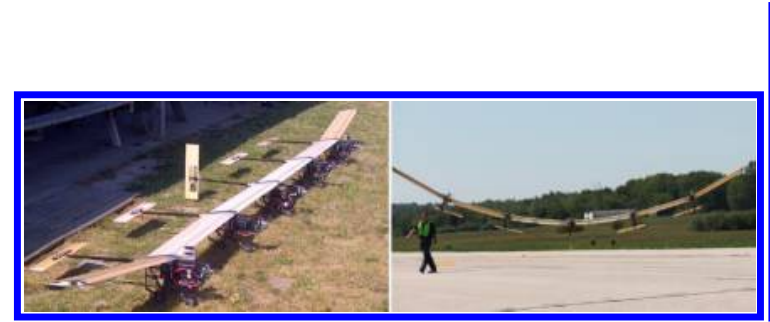

(a) Flight testing platform X-HALE

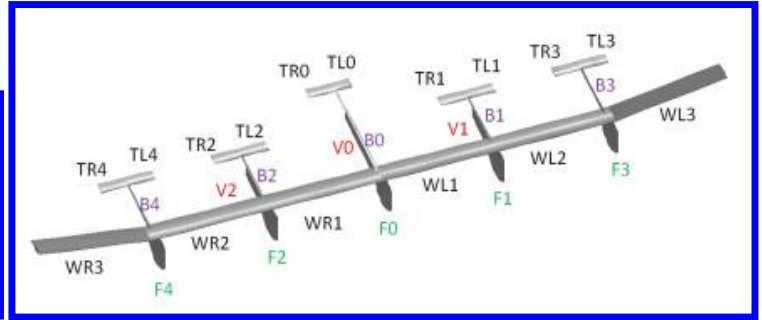

(b) Schematic CAD view of X-HALE

Figure 1: Illustration X-HALE aircraft, developed at the University of Michigan

with X-HALE. Besides linear analytical models and a nonlinear simulation environment, X-HALE exists as a real demonstration aircraft (cf. Fig. 1(a)). Therefore X-HALE is most suitable for the development and demonstration of new approaches especially of control laws that have to consider the flexibility of an aircraft. Linear models are used for the control law design, the nonlinear simulation for the validation of the controllers and the real aircraft for the demonstration under real flight conditions. Therefore X-HALE was selected as reference aircraft.

XHALE consists of six one meter wing sections as well as five pods, tails, engines and booms. The four inboard sections are modelled using two flexible elements, while outboard dihedrals are modelled using four flexible elements. Tails are modelled using two rigid elements. Booms, pods and tails are modelled by rigid elements. The lifting surfaces, wing sections, fins, tails and pods are modelled by the lifting surface method. Booms are modelled as non-lifting surfaces. The aircraft is illustrated in Fig. 1(b). For control law design, X-HALE is trimmed at an airspeed of $14 \frac{\mathrm{m}}{\mathrm{s}}$ in an altitude of $30 \mathrm{~m}$. An analytical linearized model as well as a numerical linearized model (via numerical perturbation of the states) is available for this flight condition. Additionally two other linear models for the same altitude but with reference airspeeds of $12 \frac{\mathrm{m}}{\mathrm{s}}$ and $16 \frac{\mathrm{m}}{\mathrm{s}}$ are provided by U-M. The linearized state-space model

$$
\begin{gathered}
\dot{\mathbf{x}}(t)=\mathbf{A x}(t)+\mathbf{B u}(t) \\
\mathbf{y}(t)=\mathbf{C x}(t)
\end{gathered}
$$

contains 340 states. Eight states represent the flight mechanics with velocities in all three body axis, three body rotational rates, pitch and roll angle. For structural deformations 128 states represents the strains and their derivatives. The model contains 204 aerodynamic states with 6 inflow states associated with each of the 38 lifting surfaces. It should be noted that the complete linear model contains 344 states. The four additional states are the three dimensional position and the azimuth (yaw angle). Because those states represent the navigation and follow by integration of the other states, those four states are removed for the innerloop control law design. For the outer loop those additional states are considered. The input variables are four elevators

$$
\mathbf{u}_{\eta}=\left[\eta_{T R 2}, \eta_{T R 4}, \eta_{T L 1}, \eta_{T L 3}\right]^{T},
$$

one aileron pair

$$
u_{\xi}=\xi
$$

and all five engines

$$
\mathbf{u}_{F}=\left[F_{F 0}, F_{F 2}, F_{F 4}, F_{F 1}, F_{F 3}\right]^{T} .
$$

can be used. For the output equation in Eq. 1 the flight mechanic states of roll, pitch and yaw rate as well as the structural strains $\kappa_{x}$ and $\kappa_{y}$ are used for the inner loop design. In the outer loop the design the Euler angles (yaw, pitch and roll angle), the sideslip angle and the velocity are used as additional flight mechanic parameters. It has to be notice that the velocity represents the body fixed velocity in flight direction. The airspeed is not available. The sideslip angle is computed as quotient of the velocity in right wing direction and the flight path velocity.

For the controller design in the frequency domain, Eq. 1 (assuming that the feedforward matrix is equals 
to zero) is transformed by the Laplace transformation

$$
\begin{gathered}
s \hat{\mathbf{x}}(s)=\mathbf{A} \hat{\mathbf{x}}(s)+\mathbf{B} \hat{\mathbf{u}}(s) \\
\hat{\mathbf{y}}(s)=\mathbf{C} \hat{\mathbf{x}}(s)
\end{gathered}
$$

Hence, the transfer function of the plant is determined by

$$
\mathbf{G}(s)=\hat{\mathbf{y}}(s) \hat{\mathbf{u}}^{-1}(s)=\mathbf{C}[s \mathbf{I}-\mathbf{A}]^{-1} \mathbf{B} .
$$

Relating to the analytical computed linearized model in trimmed conditions, one complex conjugated eigenvalue of the plant has a positive real part . This unstable motion can be assigned the Dutch roll. All other eigenvalues are in the left side of the complex plane. Because the mass distribution is asymmetric, there exist a strong counpling between lateral and longitudinal motion.

A detailed description of the analytical models is given in a papers of Patil and Hodges ${ }^{11}$ and about the nonlinear simulation environment UM/NAST in Shearer, Cesnik ${ }^{13}$ and Su, Cesnik. ${ }^{17}$

\section{Control Law Design}

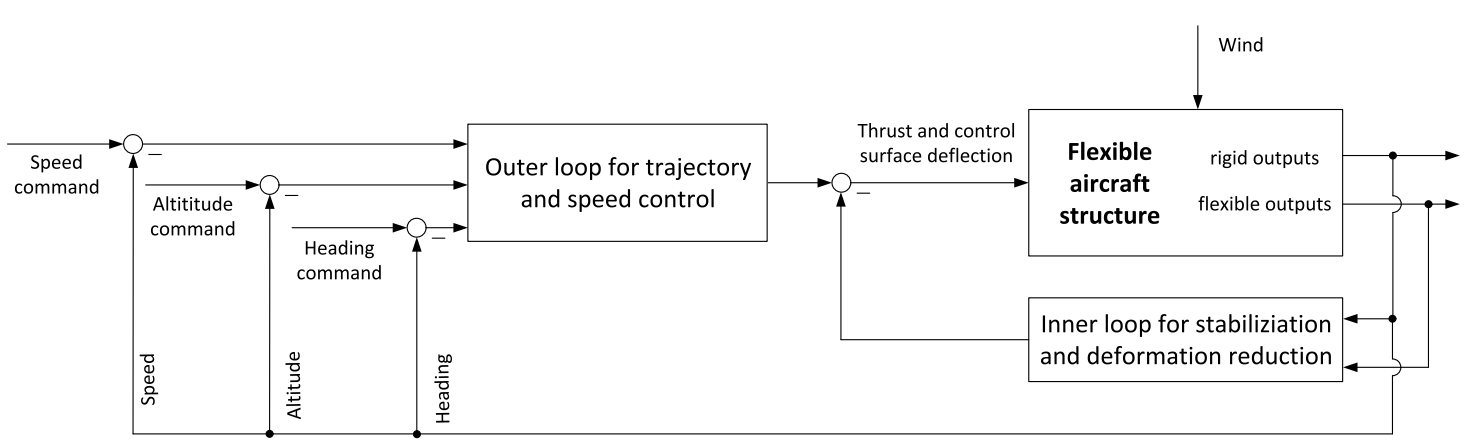

Figure 2: Control law structure for flexible aircraft structures

The flight control system shall ensure trajectory control. A cascaded control law structure is selected as illustrated in Fig. 2. The inner loop is used to minimize deviation from the trim deformation, as well as to damp out structural rates, in order to reduce the influence of the structural motion from the flight rigid body states. The inner-loop dynamics shall have a high bandwidth to assure sufficient separation from the outer-loop dynamics. The tasks of the outer loops are to acquire airspeed, altitude, and azimuth. The controlled system shall be robust against model uncertainties (time delay, parameter variations, and unmodelled nonlinear dynamics). The requirments of the flight control system and synthesis of the inner loops and outer loops is described in the following subsections.

\section{A. Requirements in control law design}

The main requirement for the control laws is stabilization of the aircraft. As a consequence, the control law has to stabilize the unstable flight dynamic motion. The control laws that are designed with linear models, shall be robust against non-modelled nonlinear dynamic behaviour. The inner loops shall compensate structural motions with a high bandwidth to ensure an optimal shape of the aircraft and provide the outerloop a nearly rigid aircraft. Mathematically the maximum deviation in strains relating to the trimmed conditions is defined with 0.05 . Furthermore the roll and pitch motion shall be damped. The maximum allowable roll rate around all axes is defined with $5 \frac{1^{\circ}}{\mathrm{s}}$. The time delay of the control system that is assumed with $50 \mathrm{~ms}$ shall be considered in the design process. Furthermore the bandwidth of the actuators (elevators and aileron) is below $10 \mathrm{~Hz}$ and the maximum deflection limit is $10^{\circ}$. A stall flight condition, indicated by a high pitch angle (angle of attack measurement is not available), has to be avoided. The outer loop speed controller shall achieve speed tracking and holding. Especially the maximum drop in airspeed for a climb shall be 
minimized. The sideslip controller shall hold sideslip equals to zero to avoid additional drag. The altitude and azimuth tracking shall be achieved with minimal overshoot and in an adequate time. Additionally the control law shall compensate gusts.

\section{B. Inner Loop}

The objective of the inner loops is the stabilization of the system and damping of rigid-body flight mechanical rotational rates and structural deformation although the dynamic behaviour is nonlinear. To achieve those goals, frequency domain control techniques like $\mathcal{H}_{2}, \mathcal{H}_{\infty}$ and robust $\mathcal{H}_{\infty}$ are used. Those methods can design control laws with or without considering uncertainties. Each method requires design criteria weights. Those weights can be directly derived from the requirements. In contrast to the classical LQG method, weights are only required for the output variables and not for all states.

For applying the methods in the frequency domain, the plant has to be normalized to the maximum input signal $u_{\max , i}$ and the maximum output signal (that is equal to the maximum error for the inner loop) $y_{\max , j} .{ }^{14}$ The maxima for every input $i$ and every output $j$ are collected in two diagonal matrices with

$$
\mathbf{D}_{u}=\operatorname{diag}\left(u_{\max , i}\right) \quad \text { and } \quad \mathbf{D}_{e}=\operatorname{diag}\left(y_{\max , j}\right) .
$$

Because only the aerodynamic control surfaces are used for the inner loop, the maximum input signal is for each input equal to $10^{\circ}$. For the indices $j=1, \ldots, 32$ (representing the strains) the maximum output signal is set to 0.05 and for $j=33,34,35$ the maximum output is defined with $5 \frac{1^{\circ}}{\mathrm{s}}$. The normalized plant $\mathbf{G}_{n}$ is computed with

$$
\mathbf{G}_{n}=\mathbf{D}_{e}^{-1} \mathbf{G} \mathbf{D}_{u} .
$$

This normalization has to consider for the controller $\mathbf{K}_{n}$ that is computed by frequency domain control techniques. This controller is also normalized and has to be retransformed by

$$
\mathbf{K}=\mathbf{D}_{u}^{-1} \mathbf{K}_{n}
$$

to a controller that can be used in simulations with non-normalized plant and in nonlinear simulation.

1. Multivariable control law design in the frequency domain

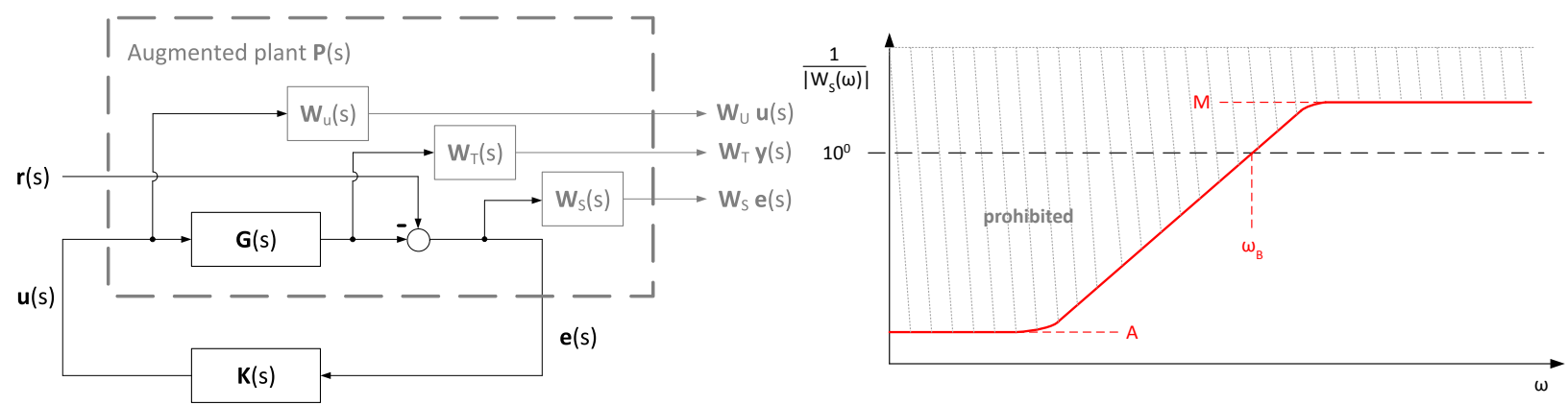

Figure 3: Augmented plant with exogenous outputs and input

Figure 4: Effect of the weight $W_{S}$ to the sensitivity $S$

The $\mathcal{H}_{2}$ control law design is a multivariable control law design technique in the frequency domain. It is based on loop shaping of the sensitivity $S$, complementary sensitivity $T$ as well as control surface activity. The plant is extended to an augmented plant with three additional exogenuous outputs

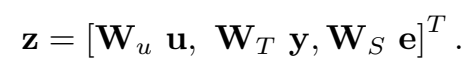

and one exogenuous input

$$
\mathbf{w}=\mathbf{r}
$$

as illustrated in Fig. 3. ${ }^{14}$ The exogenous outputs are products of the control variables, the output variables and the error with weights to shape the the output activity, complementary sensitivity and sensitivity. The 
goal of the $\mathcal{H}_{2}$ loop shaping is to find a controller that stabilizes the closed-loop system and minimizes the value

$$
V=\left\|\mathbf{W}_{u} \mathbf{u}\right\|_{2}^{2}+\left\|\mathbf{W}_{T} \mathbf{y}\right\|_{2}^{2}+\left\|\mathbf{W}_{S} \mathbf{e}\right\|_{2}^{2} .
$$

That is equal to a LQR problem. Due to the relation

$$
S=1-T
$$

only the weight for the sensitivity and control surface activity has to be defined in the controller design. According to Skogestad, ${ }^{14}$ the weight for the sensitivity is defined by

$$
W_{S}=\frac{\frac{s}{M}+\omega_{B}}{s+\omega_{B} A},
$$

where $\omega_{B}$ is the bandwidth, $A$ the lower bound and $M$ the upper bound for the sensitivity. The effect of the weight $W_{S}$ to the sensitivity $S$ is illustrated in Fig. 4 . According to the Bode Integral and Stein, ${ }^{15}$ there are limitations for the bandwidth. The upper bound of the bandwidth depends on zeros in the right hand side of the complex plane and the time delay $\tau$ with

$$
\omega_{B} \leq \frac{z}{2} \quad \text { and } \quad \omega_{B} \leq \frac{1}{\tau}
$$

where $z$ is the smallest real part of positive zeros. The lower bound of the bandwidth depends on the largest real part of unstable plant poles $p$ and is given with

$$
\omega_{B} \geq 2 p
$$

The plant has an unstable complex conjugative pole with a real part of $p=0.14$. Regarding the time delay, an overall time delay of $\tau=50 \mathrm{~ms}$ is assumed for the digital flight control system. As a consequence the bandwidth has to be within the interval

$$
\omega_{B} \in[2 \pi \cdot 0.045,2 \pi \cdot 3.1831] .
$$

Regarding to Skogestad ${ }^{14}$ and preliminary design studies, the parameter $A$ and $M$ in Eq. 14 are selected as

$$
M=1.25 \quad, \quad A_{\text {strains }}=0.4 \quad \text { and } \quad A_{p, q, r}=0.1 .
$$

The inner loops have to be much faster than the outer loops. The higher the bandwidth of the closed loop system, the faster the system reacts to disturbances. Hence, the bandwidth for the weight $W_{S}$ is selected with $\omega_{B}=2 \pi \cdot 3$ at the upper bound. For the control law, $n=35$ inputs (measurement variables) are available. Hence the matrix $\mathbf{W}_{S}$ has the dimension $35 \times 35$ and have only values along the main diagonal. Since the parameter $A$ differs for strains and rotations rates, the first 33 main diagonal elements of the matrix $\mathbf{W}_{S}$ has the form

$$
\mathbf{W}_{S}=\operatorname{diag}\left\{w_{s, 1} \ldots, w_{s, n}\right\} \quad \text { with } \quad w_{s, i}=\frac{\frac{s}{1.25}+20}{s+8} \quad \text { and } \quad i=1, \ldots, 32 .
$$

The roation rates are weighted with

$$
\mathbf{W}_{S}=\operatorname{diag}\left\{w_{s, 33} \ldots, w_{s, n}\right\} \quad \text { with } \quad w_{s, i}=\frac{\frac{s}{1.25}+20}{s+2} \quad \text { and } \quad i=33, \ldots, 35 .
$$

For the weight of the control activity a high pass filter with the transfer function

$$
w_{u}=\frac{T_{D} s}{T_{D} s+1} \quad \text { with } \quad T_{D}=\frac{1}{\omega_{C}}
$$

is used. This weight ensures that the control inputs are used until the frequency $\omega_{C}$. For the $\mathcal{H}_{2}$ inner loop, only the aerodynamic control surfaces are used. Hence the matrix $\mathbf{W}_{U}$ is a diagonal matrix

$$
\mathbf{W}_{U}=\operatorname{diag}\left(w_{u}\right)
$$


and has the dimension $5 \times 5$. Therefore the cut-off frequency $\omega_{C}$ is selected with $10 \mathrm{~Hz}$.

For the $\mathcal{H}_{2}$ control law design in MATLAB, the commands sysic for the design of the augmented plant and h2sync for the controller synthesis are used. With the desired weight and the plant, all required information for the control law design are available.

The $\mathcal{H}_{\infty}$ control law design method is similar to the $\mathcal{H}_{2}$ controller synthesis. Differences are in the minimization method and in the use of uncertainties. In $\mathcal{H}_{\infty}$ control law design, the control law is chosen such that

$$
\left\|\mathbf{W}_{u} \mathbf{u}\right\|_{\infty} \leq 1 \quad, \quad\left\|\mathbf{W}_{T} \mathbf{e}\right\|_{\infty} \leq 1 \quad, \quad\left\|\mathbf{W}_{S} \mathbf{e}\right\|_{\infty} \leq 1
$$

is fulfilled. Furthermore the $\mathcal{H}_{\infty}$ approach is suitable for considering of uncertainties. That is not the case in $\mathcal{H}_{2}$ synthesis. The implementation in MATLAB is similar to the $\mathcal{H}_{2}$ synthesis. An augmented plant is defined and the synthesis is performed with the command hinfsyn.

\section{Considering the uncertainties}

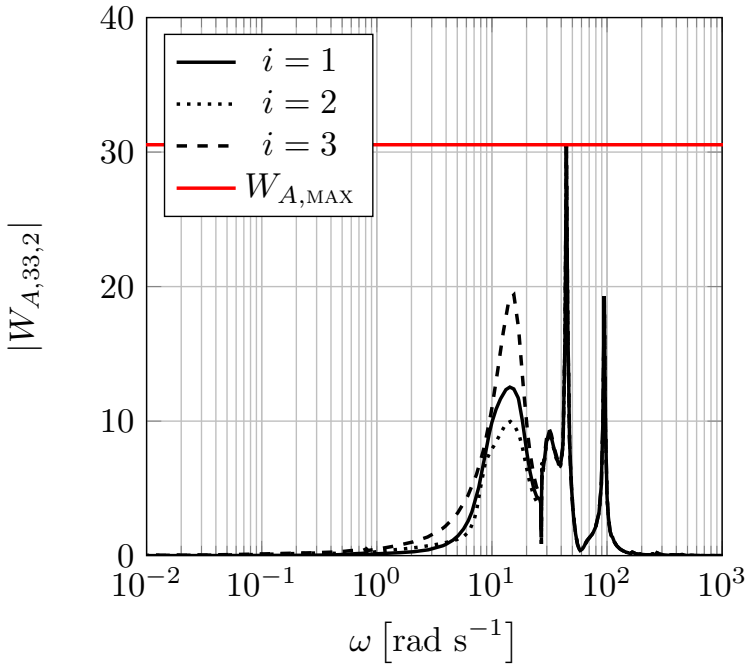

(a) Transfer function from $\eta_{T R 4}$ to $p$

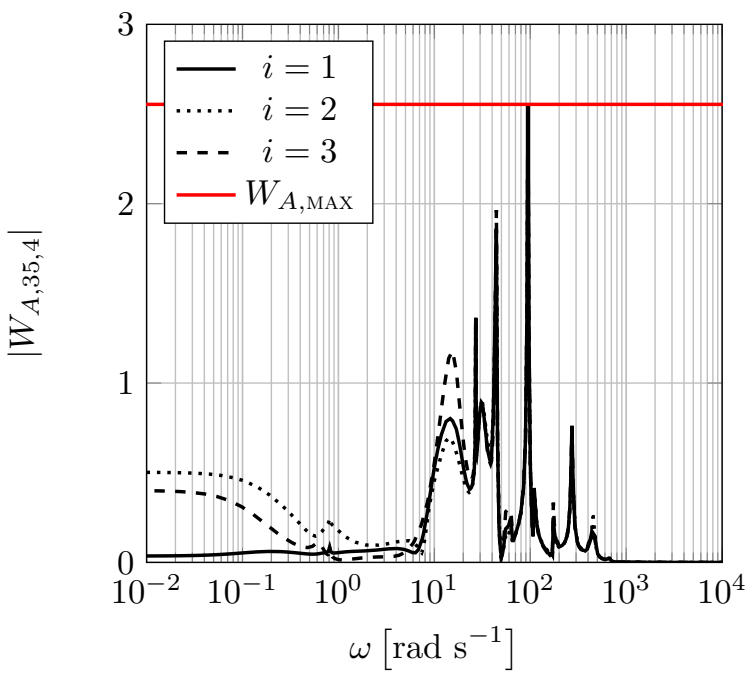

(c) Transfer function from $\eta_{T L 3}$ to $r$

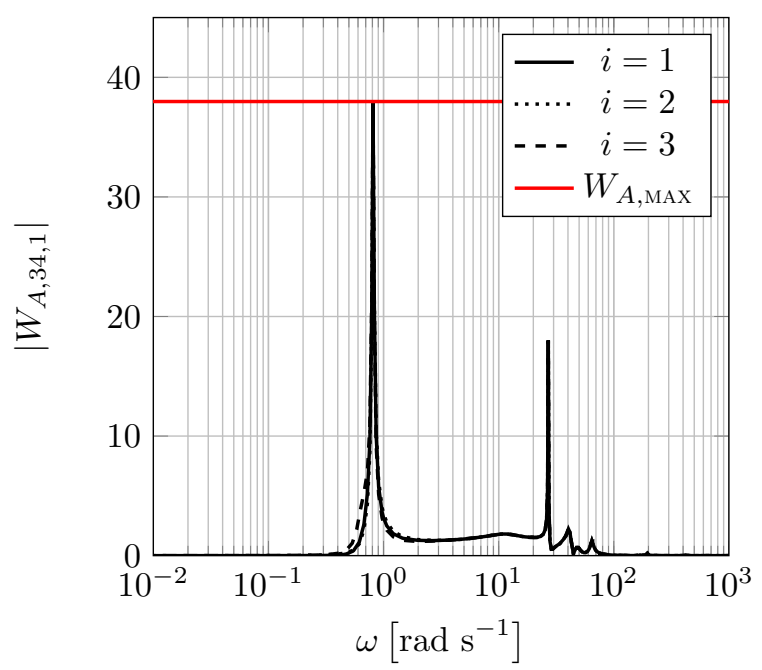

(b) Transfer function from $\eta_{T R 2}$ to $q$

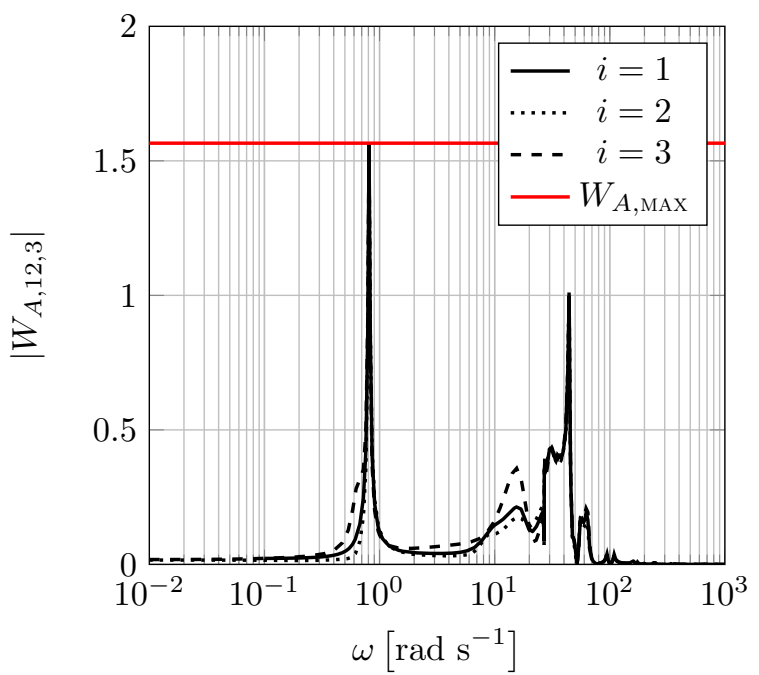

(d) Transfer function from $\eta_{T L 1}$ to $\kappa_{y}$ at the right wing tip

Figure 5: Illustration of selected uncertainties as a function of the frequency between the nominal plant and the uncertain models and the maximum scalar entry of the matrix $\mathbf{W}_{A}$ 
Nonlinearities of the aircraft structural dynamics shall be described as uncertainties. It is assumed that different deformations lead to changes in the eigenvalues of the linearized system. This effect is obviously for a pendulum. The pendulum is deflected to different angular displacement and the steady state is achieved by an external moment. Now a linearization around the steady state is carried out and the damping of the pendulum is a function of the steady angular displacement. Transfering this method to a highly flexible aircraft, trimming different load cases leads to different (nonlinear) deformations and hence different eigenvalues. ${ }^{16}$ Those differences can be summed up as uncertainties of the plant. For the current use case, linear models for different load cases are not available. Nevertheless analytically computed linear models at different airspeeds are avialable that are assumed as uncertain models $\mathbf{G}_{\mathrm{P}, i}$. The index $i=1$ represents the analytic computed linearized model for the airspeed of $14 \frac{\mathrm{m}}{\mathrm{s}}$. The index $i=2$ belongs to the model of $12 \frac{\mathrm{m}}{\mathrm{s}}$ and the index $i=3$ to the model of $16 \frac{\mathrm{m}}{\mathrm{s}}$. The numerical linearized model of X-HALE is considered as nominal plant G. With this nominal model and the family of uncertain models, an unstructured uncertainty matrix $\mathbf{W}_{\mathrm{A}}$ can be computed with

$$
\mathbf{W}_{\mathrm{A}, i}=\mathbf{G}-\mathbf{G}_{\mathrm{P}, i}
$$

for each uncertain model. After all iterations for every input-output combinaton, the uncertainty weight matrix must be equal or greater than the maximal singular values of the computed differences for all frequencies and all considered models. Often scalar values are used for $\mathbf{W}_{A}$, but also transfer functions are possible. ${ }^{14}$ The advantage of scalar values is that the order of the control law is not increased, if the $\mathcal{H}_{\infty}$ design method with consideration of uncertainties is used. For the roll, pitch and yaw rate as well as the strain $\kappa_{y}$ at the right wing, the calculation of the uncertainties is illustrated in Fig. 5 for selected input variables. It can be seen that the dynamic behaviour for the lateral states differs for every uncertain plant while the dynamic behaviour in the longitudinal motions is similar for plants with different uncertainties. Especially for the rigid body states, the uncertainties have very high values. This indicates that nominal model and uncertain models differ strongly in those states. The deviations of the structural states are in an acceptable range.

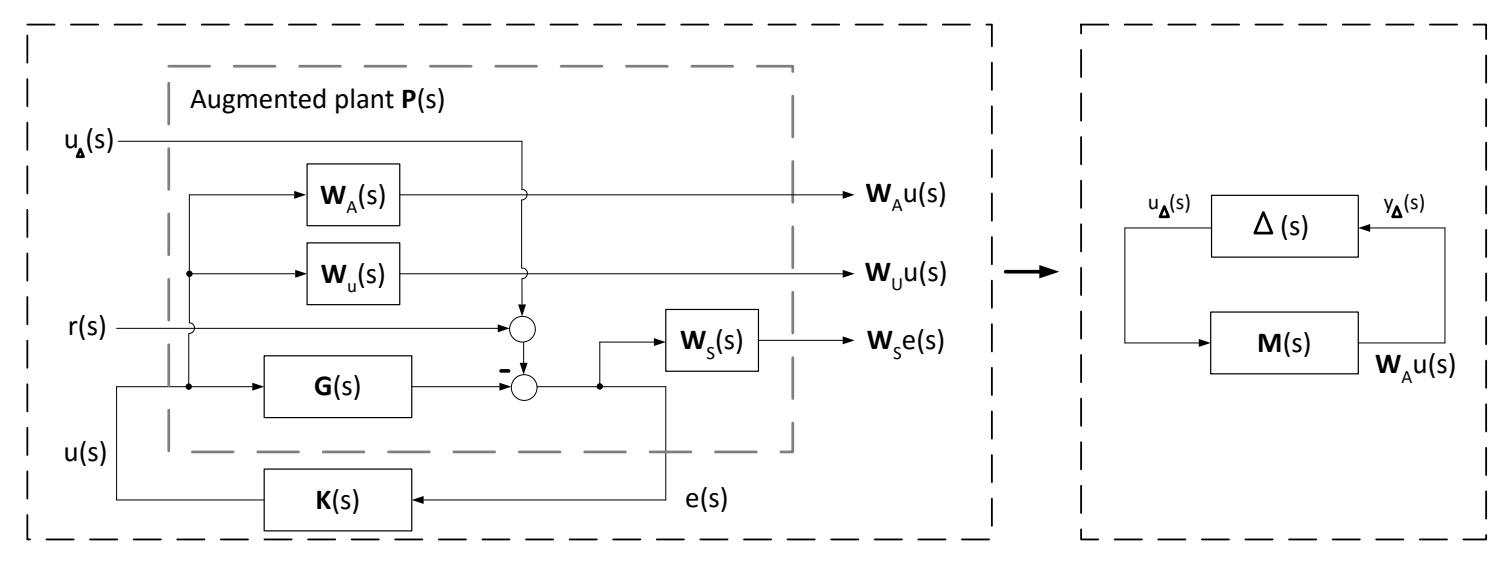

Figure 6: Transformation of the augmented plant with uncertainties to the $M \Delta$ structure

For the evaluation of robust stability, the uncertain plant is formulated in a $M \Delta$ structure. ${ }^{14}$ Robust stability for a $M \Delta$ system for all allowed perturbations is given for a nominal stable $\mathbf{M}$ and $\boldsymbol{\Delta}$ if and only if, the structured singular $\mu$ value of $\mathbf{M}$ is smaller than one for all frequency

$$
\mu(\mathbf{M}(j \omega))<1 \quad \text { for all } \omega
$$

for all $\Delta \in \Pi$, where $\Pi$ is the set of allowable perturbations. ${ }^{14}$ Robust stability can be evaluated by the MatLAB command robuststab. The state space system is formed into a $M \Delta$ system by a lower fractional transformation of the augmented plant and the transfer function $\boldsymbol{\Delta}$ with the help of the MATLAB command lft. The transformation from the augmented plant with uncertainties to the $M \Delta$ structure is illustrated in Fig. 6. 


\section{Model Reduction}

The disadvantage of control law designs with the $\mathcal{H}_{2}$ and $\mathcal{H}_{\infty}$ method is the high order of the control law. The order is the sum of the plant order and the order of the weights. As a consequence the control laws possess an order of 380 (order 340 of the plant, order 35 of the weights for the measurement variables and order 5 of the weights for the control surfaces). This order is not suited for practical applications and has to be reduced. This is achieved by balanced truncation.

The idea behind balanced truncation methods is to neglect those parts of the system that are less observable and less controllable. This leads to a system that is of lower order and retains the important dynamic behaviour of the original system. In the balanced truncation method, a state similarity transformation is applied first to balance the controllability and observability features of the system. The linear system

$$
\begin{aligned}
& \dot{\mathbf{x}}=\mathbf{A} \mathbf{x}+\mathbf{B} \mathbf{u} \\
& \mathbf{y}=\mathbf{C} \mathbf{x}+\mathbf{D} \mathbf{u}
\end{aligned}
$$

is called balanced, if solutions $\mathbf{P}$ and $\mathbf{Q}$ of the Lyapunov equations

$$
\mathbf{A} \mathbf{P}+\mathbf{P} \mathbf{A}^{T}+\mathbf{B} \mathbf{B}^{T}=\mathbf{0}
$$

and

$$
\mathbf{A}^{T} \mathbf{Q}+\mathbf{Q} \mathbf{A}+\mathbf{C}^{T} \mathbf{C}=\mathbf{0}
$$

satisfy the requirement

$$
\mathbf{P}=\mathbf{Q}=\operatorname{diag}\left\{\sigma_{1}, \sigma_{2}, \ldots, \sigma_{n}\right\} \quad \text { with } \quad \sigma_{1} \geq \sigma_{2} \geq \ldots \geq \sigma_{n},
$$

where $\sigma$ are the Hankel singular values of the system. ${ }^{8}$ Based on Eq. (27), a balanced realization of the system via state-space transformation can be computed. The balanced truncation is carried out with the MATLAB method balancmr. ${ }^{8}$

The transformation matrix is determined by the following steps. Firstly, the modified matrices $\tilde{\mathbf{P}}$ and $\tilde{\mathbf{Q}}$ are defined, depending on the order of the reduced model $k$ as

$$
\tilde{\mathbf{P}}=\tilde{\mathbf{Q}}=\operatorname{diag}\left\{\sigma_{1}, \sigma_{2}, \ldots, \sigma_{k}\right\} .
$$

In the second step, the Cholesky factors of the matrices of Eq. (30) are computed with

$$
\tilde{\mathbf{P}}=\mathbf{S}^{T} \mathbf{S} \text { and } \tilde{\mathbf{Q}}=\mathbf{R}^{T} \mathbf{R} .
$$

In the third step, the singular value decomposition of $\mathbf{S} \mathbf{R}^{T}$ is computed with

$$
\mathbf{S} \mathbf{R}^{T}=\left[\mathbf{U}_{1}, \mathbf{U}_{2}\right]\left[\begin{array}{cc}
\boldsymbol{\Sigma}_{1} & 0 \\
0 & \boldsymbol{\Sigma}_{2}
\end{array}\right]\left[\begin{array}{c}
\mathbf{V}_{1}^{T} \\
\mathbf{V}_{2}^{T}
\end{array}\right]
$$

With the transformation matrices

$$
\mathbf{W}=\mathbf{R}^{T} \mathbf{V}_{1} \boldsymbol{\Sigma}_{1}^{-\frac{1}{2}} \text { and } \mathbf{V}=\mathbf{S}^{T} \mathbf{U}_{1} \boldsymbol{\Sigma}_{1}^{-\frac{1}{2}}
$$

the matrices of the reduced system are

$$
\begin{array}{cc}
\mathbf{A}_{\text {red }}=\mathbf{W}^{T} \mathbf{A} \mathbf{V} & \mathbf{B}_{\text {red }}=\mathbf{W}^{T} \mathbf{B} \\
\mathbf{C}_{\text {red }}=\mathbf{C} \mathbf{V} & \mathbf{D}_{\text {red }}=\mathbf{D}
\end{array} .
$$

To define the minimal achievable control law order, the order of the control law was reduced from full to one by one order steps until a stop criterion was reached. The stop criterion for the $\mathcal{H}_{2}$ control law was that nominal stability of the closed loop system was required. For the robust $\mathcal{H}_{\infty}$ control law, both nominal and robust stability of the closed loop have to be fulfilled. 


\section{Outer Loops}

The inner control loops shall assure that the highly flexible aircraft structure stays quasi-steady in its reference shape. This shall allow using of classical flight control outer loops for trajectory and speed control task. Classical flight control law architectures consist of multiple cascades or loops. ${ }^{1}$ The inner loop provides damping (stability augmentation system). The next loop controls the attitude (pitch and bank angle) and the aerodynamic state (angle of attack, sideslip angle and airspeed). The next cascade is responsible for flight path control (altitude and azimuth) and the outer cascades allow full control of the flight trajectory thus supporting navigation. In this paper, a flight path control system is developed and the first, second and third inner cascades are addressed. The design of the first cascade is described in the previous sections. The inner loop requires a wash out filter for the yaw rate in order to allow turns.

\section{Extension of the Inner Loops}

The inner loops shall damp the rotational modes and the structural modes. If the aircraft performs a steady turn, a damping of the yaw rate counters the intended azimuth change in turn direction. A washout filter is required that allows a steady turn, but does not affect damping of the Dutch roll mode. The washout filter is a first-order high-pass filter with a time constant of $T_{W O}=2,5 \mathrm{~s}$ according to Brockhaus ${ }^{1}$ and Stevens. ${ }^{18}$ This leads to a filter with the transfer function

$$
F_{W a s h \text { out }}=\frac{T_{W O} s}{T_{W O} s+1} .
$$

After the yaw rate is filtered by the washout, it is used as input signal for the inner loop.

\section{Second Cascade: Atttitude and Aerodynamic State Control System}

The second cascade controls the attitude and the aerodynamic state, i.e. the the pitch and bank angle (attitude), the sideslip angle and the airspeed (aerodynamic state) have to be measured and controlled. Suitable control variables for the pitch motion (to control the pitch angle), roll motion (bank angle), yaw motion (sideslip angle) and thrust (airspeed) are chosen as a combination of the available control inputs shown in Fig. 1(b). For the pitch motion the elevators $\eta_{T R 2}$ and $\eta_{T L 1}$ are used. The roll motion is controlled with a linear combination of a positive deflection of the elevator $\eta_{T L 3}$ and a negative deflection of the elevator $\eta_{T R 4}$. The thrust is controlled by the three inner motors $F_{F 0}, F_{F 1}$ and $F_{F 2}$. The yaw motion is influenced by a linear asymmetric combination of thrust $F_{F 3}$ and $F_{F 4}$. The aileron is not used by the outer loops. The control variables of the second cascade are calculated by

$$
\begin{array}{ll}
u_{1, \text { Pitch }} & =\eta_{T R 2}+\eta_{T L 1} \\
u_{2, \text { Thrust }} & =F_{F 0}+F_{F 1}+F_{F 2} \\
u_{3, \text { Roll }} & =\eta_{T L 3}-\eta_{T R 4} \\
u_{4, \text { Yaw }} & =F_{F 3}-F_{F 4}
\end{array} .
$$

For X-HALE lateral and longitudinal motion are coupled. As this behavior is undesirable, a decoupling controller is used to decouple lateral and longitudinal states. This has the advantages that a sequential loop design can be applied for the second as well as the third cascade. The decoupling control law is a steady gain matrix without any dynamics. This leaves some coupling in the high frequency dynamics, but it decouples the steady states. Preliminary investigation showed that a steady-gain decoupling controller is sufficient. Furthermore the decoupler is used for steady turns. If the aircraft is in a turn, the lift vectors also banks and hence less lift to compensate the weight is available. A change in the bank angle has to lead to an increasing pitch angle. If the aircraft increases the pitch angle, a drop in the velocity occurs. This effect is also compensated by the decoupler.

The goal of pitch angle control law is to increase the damping of the phugoid. Therefore just a steady gain is used for the control law. The gain is determined with the root locus method of the transfer function from $u_{1, P i t c h}$ to the pitch angle. Therefore the transfer function was reduced with balanced truncation to forth order. To speed up the behaviour for tracking, a feedforward controller is used. The command pitch angle is limit to $\pm 10^{\circ}$. The complete design of the pitch control law is similar to traditional approaches as described by Brockhaus ${ }^{1}$ and Stevens. ${ }^{18}$ 
Since there is no natural integration in the transfer function from thrust to airspeed, a proportionalintegral (PI) controller is used. Based on the second-order reduced transfer function from thrust to flight path velocity, this controller is designed to reject fast disturbance in the flight path velocity. A feedforward element is used to achieve fast tracking. The design method of this controller is similar to the method described by Stevens ${ }^{18}$ that is used for rigid body aircraft.

The bank angle command controller leads to an improvement in the roll motion due to an influence of the roll time constant. Hence, in the decoupled uncontrolled system the roll motion is not an aperiodic motion, rather an coupled oscillation with spiral mode. The root locus method can be used to split the oscillation into two aperiodic motions (spiral and roll mode). To apply the method, the transfer function from $u_{3, \text { Roll }}$ to the roll angle is reduced to fourth order. A steady gain is estimated with the root locus method. Due to the third cascade (azimuth control), an integration part in the control law is not required. The command bank angle is limit to $\pm 15^{\circ}$. Similar to the pitch and speed control law, a feedforward element is used to speed up the reaction of the aircraft.

The sideslip control law shall reduce the sideslip angle and increase the damping of the Dutch roll. Based on the forth-order reduced transfer function from $u_{4, Y a w}$ to the sideslip angle, a steady gain is determined by applying the root locus method. This is similar to classical approaches. With the sideslip control law, all components of the second cascade are determined. The block diagram of the second cascade is illustrated in Fig. 7(b).

3. Third Cascade: Altitude and Azimuth Control System

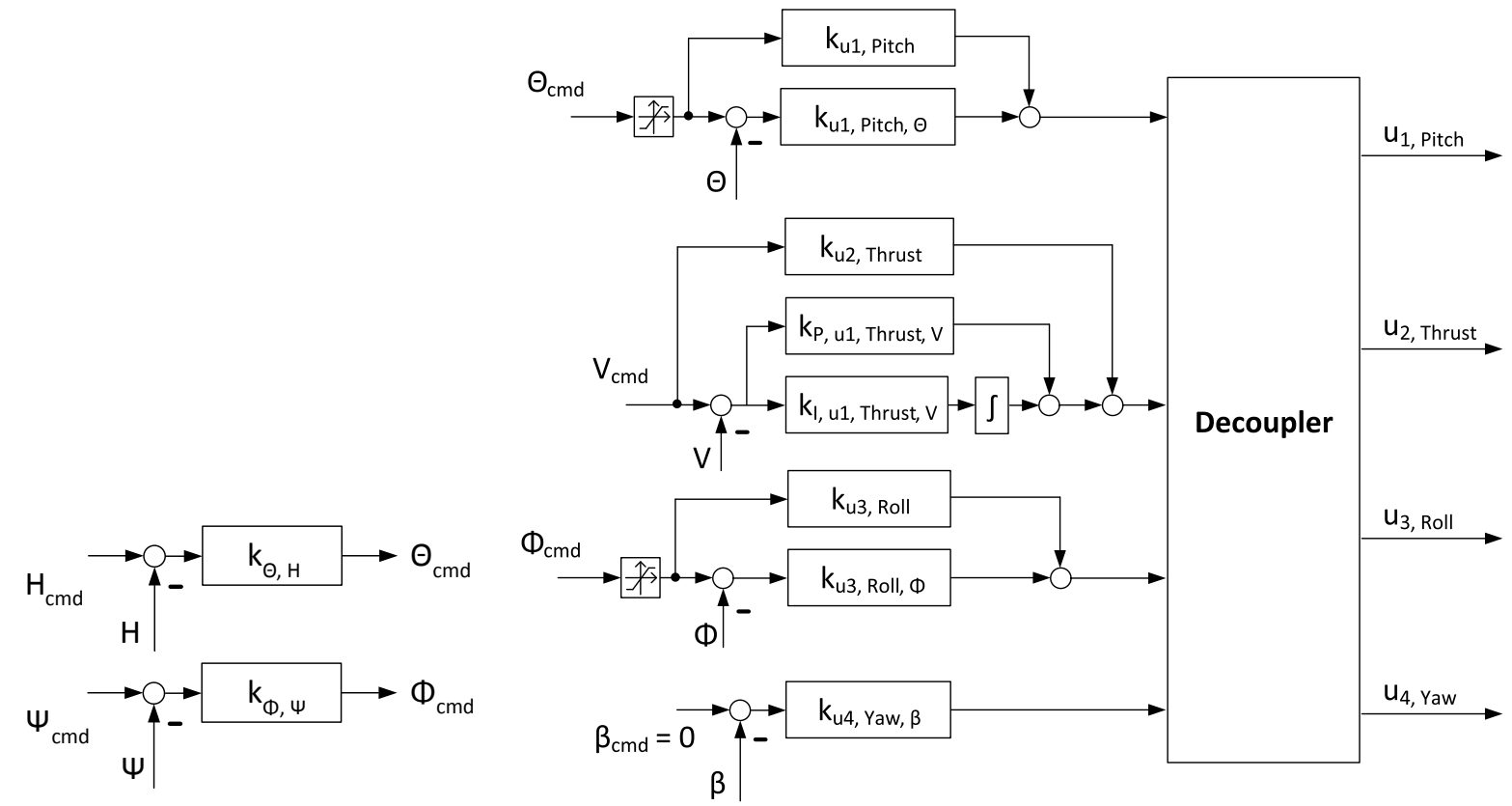

(a) Third cascade for altitude and azimuth conntrol

(b) Second cascade for pitch, bank, sideslip and airspeed control

Figure 7: Illustration of block diagram for the trajectory control outer loops

The third cascade of the trajectory control system ensures accurate tracking of altitude and azimuth. Considering the transfer function of the pitch angle command to the altitude response and the bank angle command to azimuth response, both open loop transfer functions possess integral behaviour. Therefore no additional integrator is needed in the control law for steady state tracking. With a proportional element the fastness of the control system can be set up. To avoid oscillations, the bandwidth of the third loop has to be smaller than the one of the second cascade. The gains for the control laws are determined with root locus method. In altitude control the gain is a compromise between fast tracking and overshoot. Regarding 
the bandwidth of the second cascade, the gain of the azimuth control law is calculated for a fast reaction in azimuth tracking. The block diagram for the third cascade is illustrated in Fig. 7(a).

\section{Results}

In this section the characteristics of the inner and outer loops as well as linear and nonlinear simulation results are presented. Based on the characteristics regarding to the requirements, one inner loop control law is selected and used for the sequential loop shaping of the outer loops. The selected combination is simulated in a linear simulation with the nominal plant and in the nonlinear UM/NAST simulation environment.

\section{A. Characteristics of inner and outer loops}

Table 1: Comparison of the properties of the developed control laws

\begin{tabular}{lcccc}
\hline & $\begin{array}{c}\text { Controller } \\
\text { States }\end{array}$ & $\begin{array}{c}\text { Max. real part } \\
\text { of controller }\end{array}$ & $\begin{array}{c}\text { States } \\
\text { closed loop }\end{array}$ & $\begin{array}{c}\text { Max. real part } \\
\text { of closed loop }\end{array}$ \\
\hline $\mathcal{H}_{2}$ & 380 & -0.2587 & 720 & -0.0138 \\
$\mathcal{H}_{2}$ reduced & 32 & -0.3839 & 372 & -0.0078 \\
$\mathcal{H}_{\infty}$ & 380 & -0.2587 & 720 & -0.0138 \\
$\mathcal{H}_{\infty}$ reduced & 24 & -0.2039 & 364 & -0.0308 \\
$\mathcal{H}_{\infty}$ robust & 380 & -0.2942 & 720 & -0.0138 \\
$\mathcal{H}_{\infty}$ robust reduced & 8 & -0.5668 & 348 & -0.0146 \\
\hline
\end{tabular}

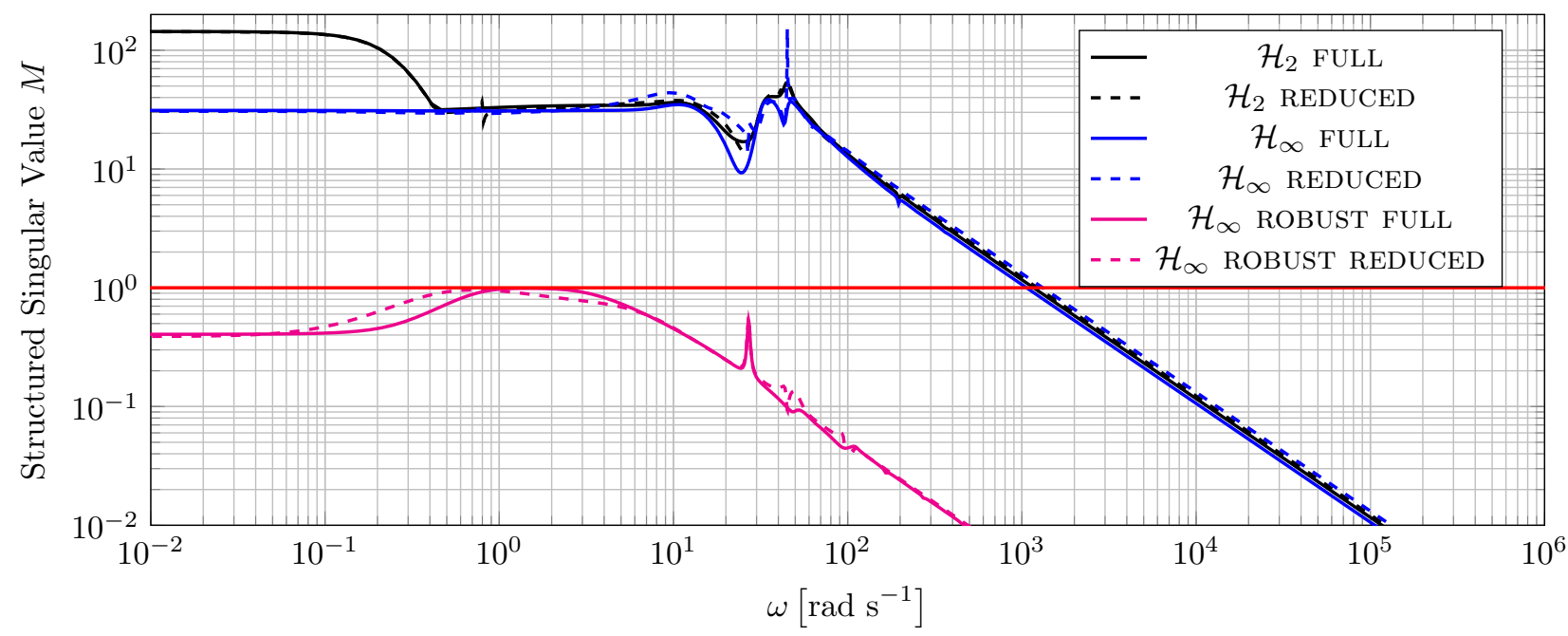

Figure 8: Evaluation of robust stability based on the structured singular value of $\mathbf{M}$ for the different innerloop controller

The synthesis of the inner loops with frequency domain design techniques was successful, but all control laws are of high order. To reduce the order of the control laws, the balanced truncation method using the stop criteria defined at section III.2.b is applied. The $\mathcal{H}_{2}$ control law can be reduced to an order of 32 , the $\mathcal{H}_{\infty}$ control law to an order of 24 and the robust $\mathcal{H}_{\infty}$ control law to an order of eight. In Tab. 1 the properties of the developed control laws are compared with each other. The maximum real part of the closed loop system is negative for all control laws. That ensures nominal stability of the closed loop system. Due to the controller reduction, the stability margin of the closed loop (distance to the imaginary axis) is decreased for the $\mathcal{H}_{2}$ and $\mathcal{H}_{\infty}$ controller. The reduced robust controller has a higher stability margin. Additionally 
to nominal stability, the developed control laws are investigated regarding to robust stability with the $M \Delta$ structure. The results are shown in Fig 8. Relating to the small gain theorem the structured singular value for $\mathbf{M}$ and all frequencies has to be smaller than one. This criterion is just satisfying for the controller that taking the uncertainties into account. All other controllers are nominal, but not robust stable.

Stability investigation of outer loop is carried out with the help of the MATLAB routine loopmargin to determine phase and gain margins as well as $-180^{\circ}$ and $0 \mathrm{~dB}$ crossover frequencies with the full transfer functions. The results for the open-loop transfer functions from the speed command to the speed response, from the sideslip command to the sideslip angle response, from the altitude command to the altitude response and from the azimuth command to the azimuth angle response are given in table 2 . The frequency response function crosses the $-180^{\circ}$ phase multiple times. The lowest value is used for the evaluation. All closed loop transfer functions fulfil the stability requirement of MIL-F-9490D ${ }^{19}$ regarding the phase angle. The gain margin of the control loops are a little bit too low. They shall be increased in a next control law iteration process.

Table 2: Stability and robustness properties for the outer loop with classical flight control approaches

\begin{tabular}{lcccc}
\hline & \multicolumn{4}{c}{ Control law for } \\
& Velocity & Sideslip & Altitude & Azimuth \\
\hline phase margin $\left[^{\circ}\right]$ & 45.31 & - & 71.27 & 81.34 \\
$0 \mathrm{~dB}$ crossover frequency $\left[\mathrm{rad} \mathrm{s}^{-1}\right]$ & 0.267 & - & 0.1044 & 0.1603 \\
gain margin [1] & 2.49 & 13.82 & 2.15 & 20.72 \\
$-180^{\circ}$ crossover frequency $\left[\mathrm{rad} \mathrm{s}^{-1}\right]$ & 0.39 & 0.46 & 0.3 & 1.43 \\
\hline
\end{tabular}

\section{B. Linear closed loop simulation}

Linear simulations are carried out with MATLAB. Every control loop is transformed into state space formulation. Based on the command inputs the third cascade computes the demand values for the second cascade. The command pitch and roll angle is bounded. Afterwards the ten output values of the second cascade are determined. Those values and the values of the inner loop controller are summed together and used as input signal for the plant. Then the plant outputs are computed and the simulation loop starts from the beginning. The complete simulation is carried out with a frequency of $400 \mathrm{~Hz}$ and the state space differential equation is solved with a fourth-order Runge-Kutta integration. Using a higher simulation step size or a simple Euler integration leads to unstable results for the closed loop. Two simulation runs are carried out.

In the first investigation, the simulation starts at an altitude of $\delta H=0 \mathrm{~m}$ and the altitude command is $\delta H_{\mathrm{cmd}}=50 \mathrm{~m}$. All other demand values are set to zero. The results of the linear simulation are illustrated in Fig. 9 to 11. Altitude tracking is achieved with no overshoot. The speed controller reacts very fast and just a small drop in the flight patch velocity occurs. Despite the zero yaw command, the aircraft begins with a yaw motion. Nevertheless the total derivation of zero azimuth is negligible. The sideslip controller works fine and only a low derivation in the sideslip occurs. As illustrated in Fig. 10 the maximum allowable pitch angle of $10^{\circ}$ is not exceeded and stall protection is achieved. Although the inner loops should damp the rotational rates and the influence of structural oscillations on the rigid body states, a $4 \mathrm{~Hz}$ oscillation is visible in the pitch and roll rate. This high frequency structural motion is also obvious in Fig. 11, but in general the strains are very low. Summarized, the designed control law achieve good performance in altitude tracking with acceptable control surface activity, but the influence of the structural motions on the flight mechanical parameter should be improved.

In a second test case, the azimuth angle command is set to $10^{\circ}$, while all other demand values are zero. The results are illustrated in Fig. 12 to 14. The commanded azimuth angle is acquired in a satisfactory time with a small overshoot. The drop in altitude and velocity is very low and acceptable. After a maximum of minus one degree, the sideslip angle reduces very fast to zero. As illustrated in Fig. 13 also in azimuth tracking an influence of structural oscillations to the rigid body states is obviously, but it dies away very fast. The pitch angle is very low and the maximum bank angle lies within an acceptable range. The structural behaviour for this autopilot mode is illustrated in Fig 14. As mentioned before, there is an extensive structural response, 

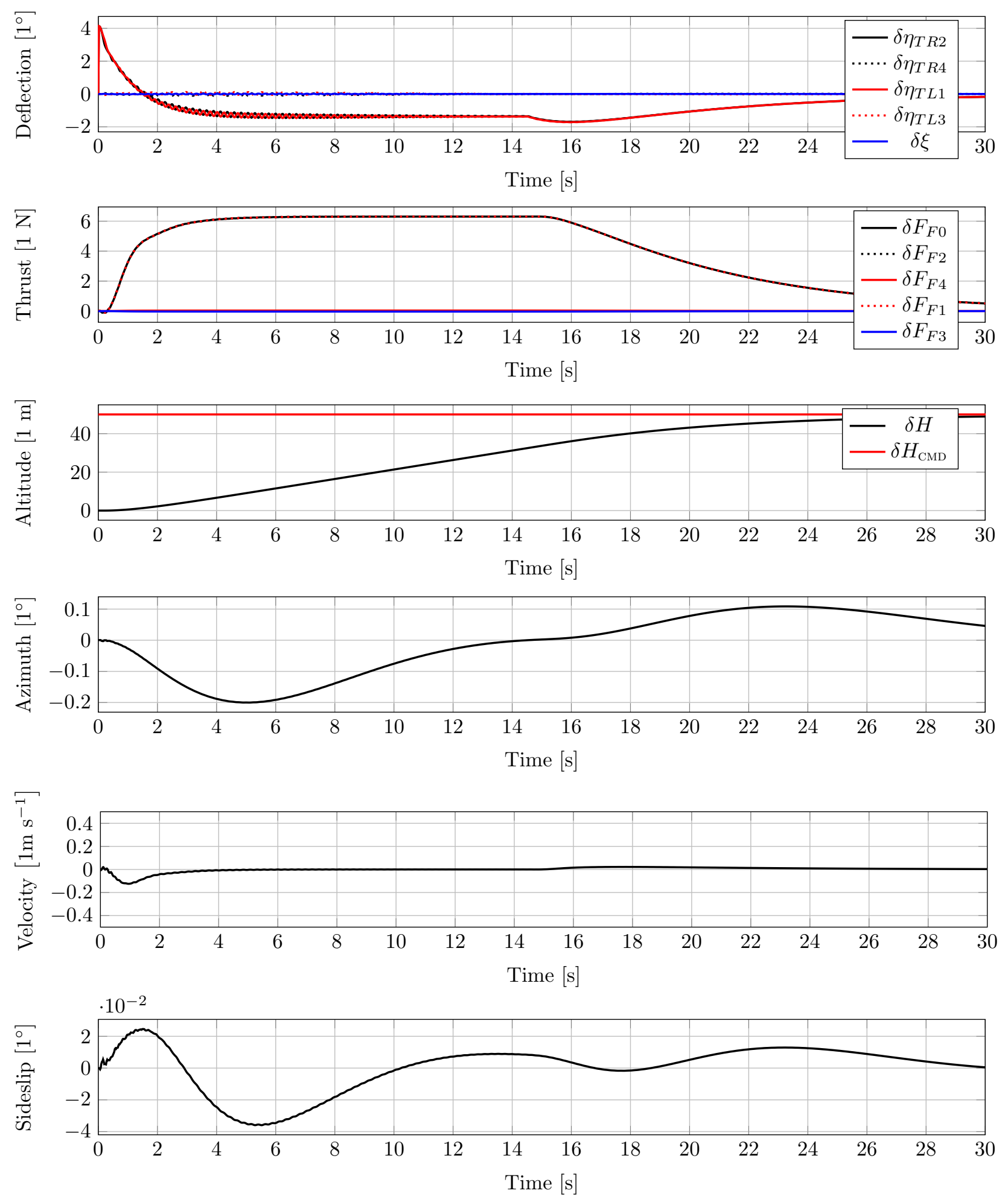

Figure 9: Illustration of control activity and flight mechanical parameters $\delta H, \delta \Psi, \delta V, \delta \beta$ for an altitude step input command (linear simulation) 

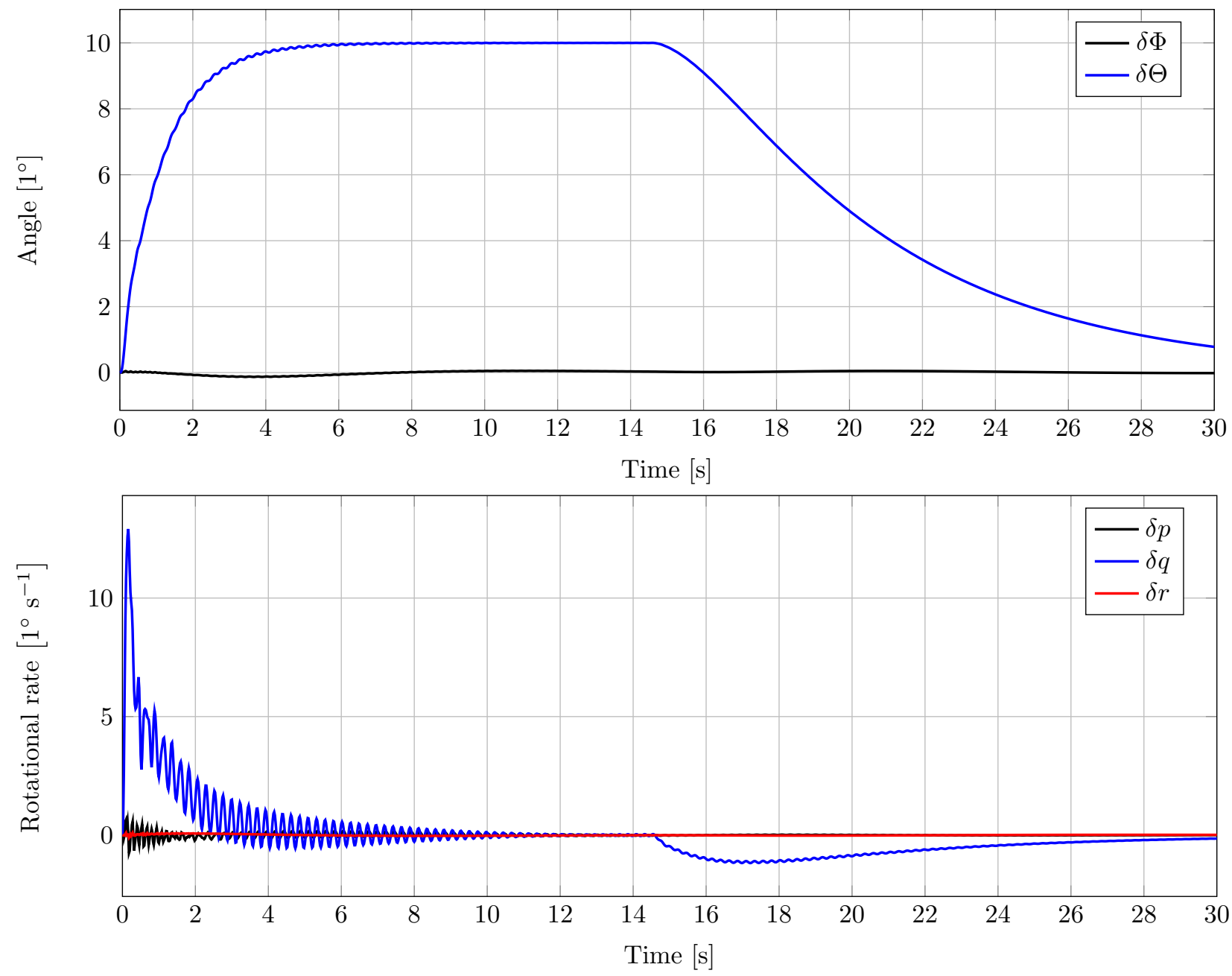

Figure 10: Illustration of pitch and roll angle as well as rotation rates around all axes for an altitude step input command (linear simulation)
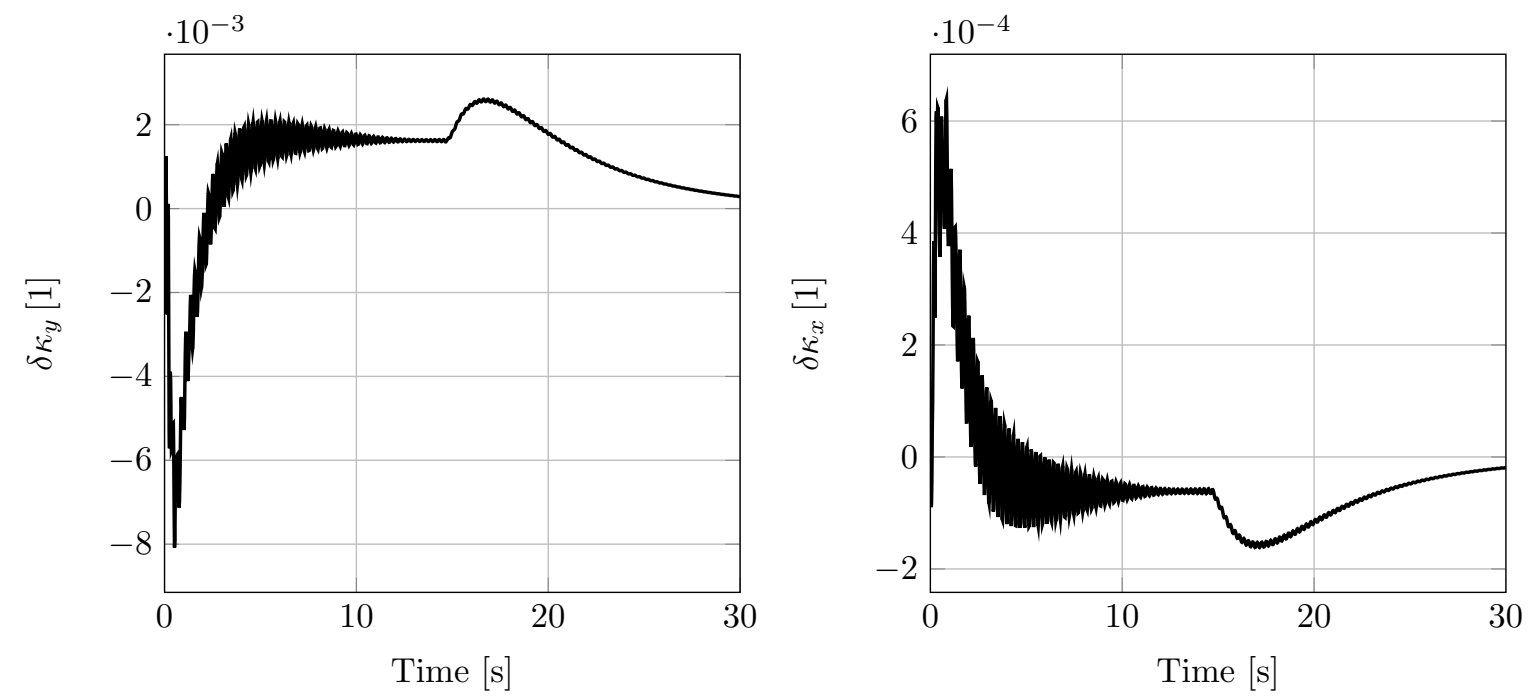

Figure 11: Illustration of curvature at the right wing tip for an altitude step input command (linear simulation) 

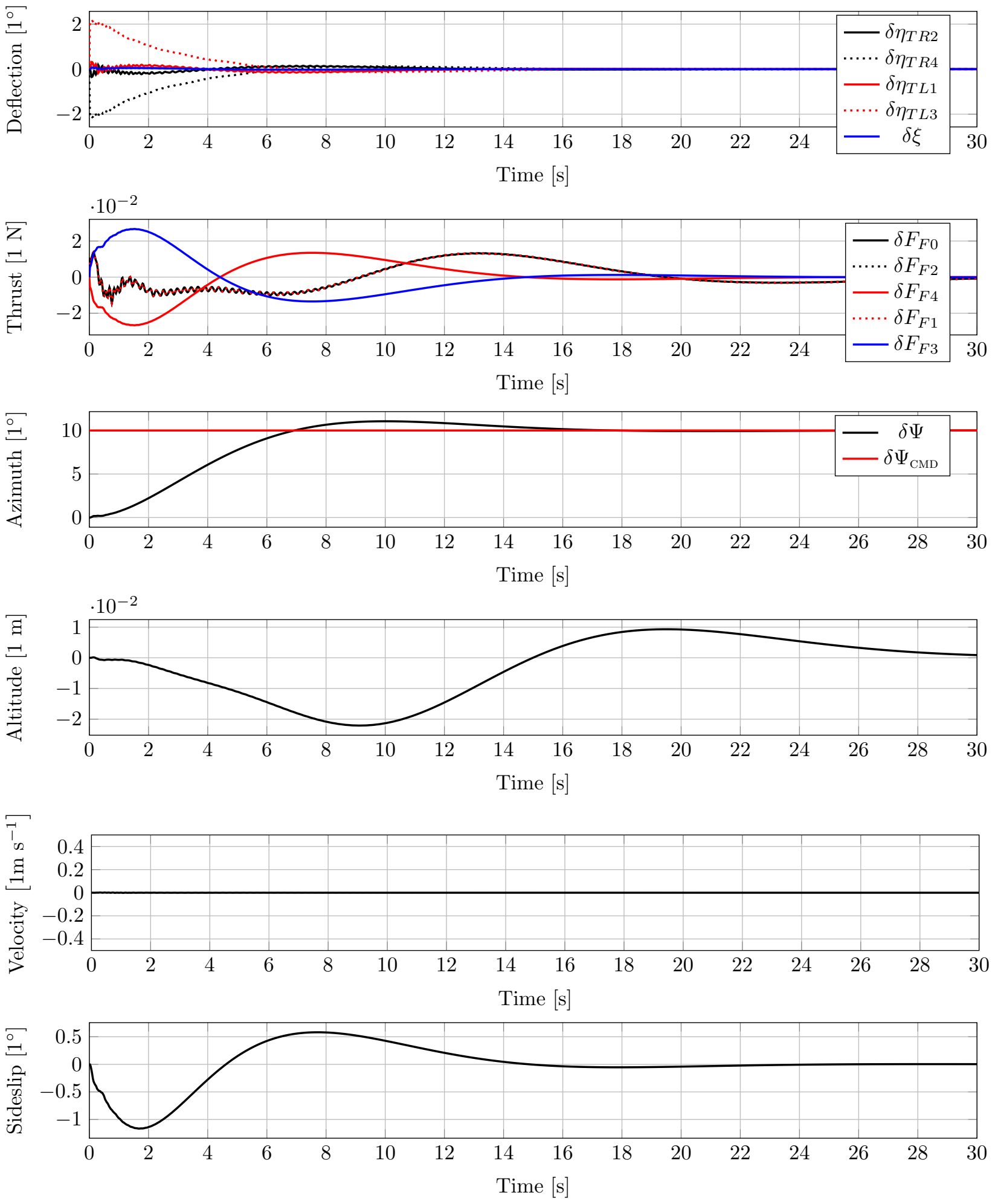

Figure 12: Illustration of control activity and flight mechanical parameter $\delta \Psi, \delta H, \delta V, \delta \beta$ for an azimuth step input command (linear simulation) 

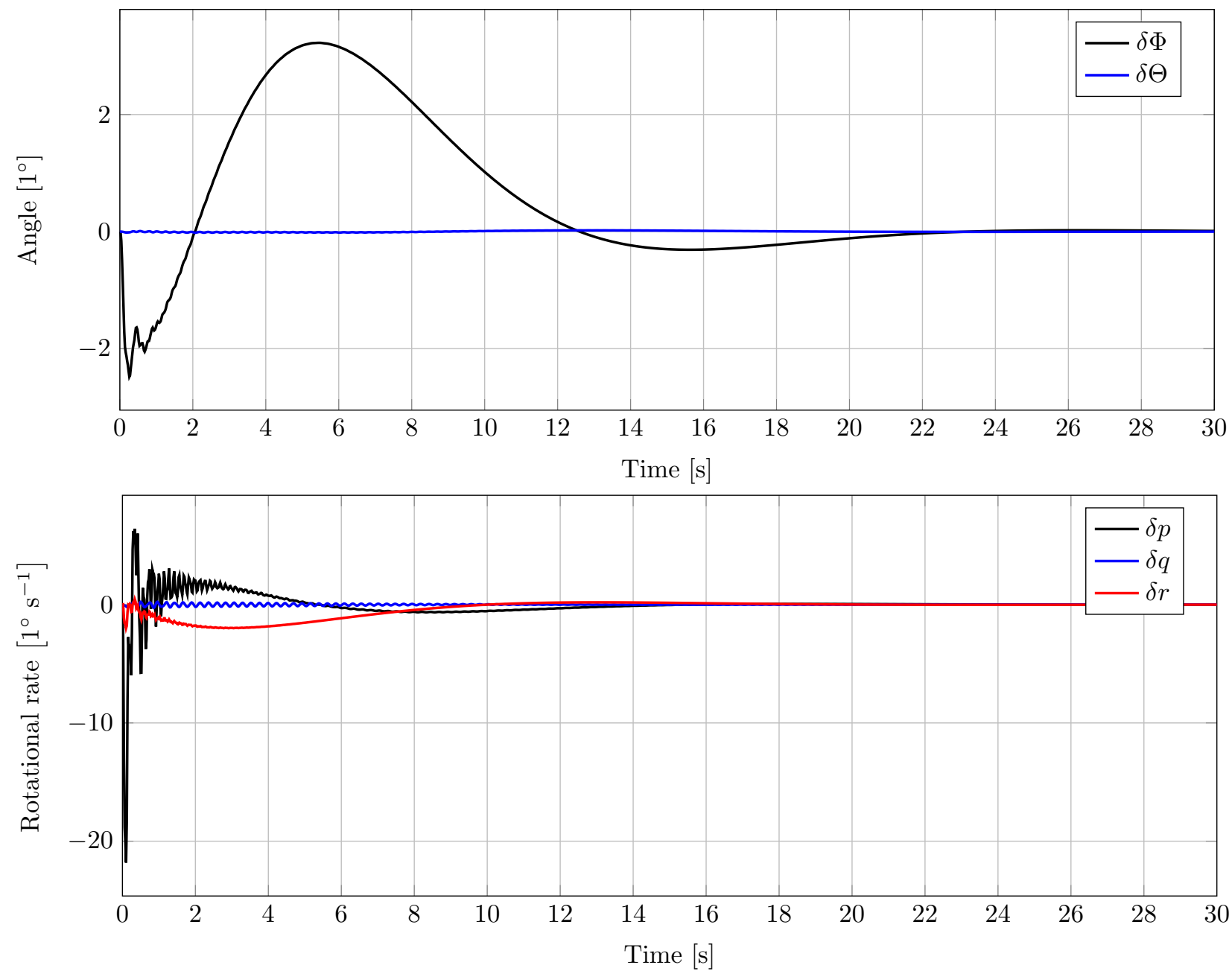

Figure 13: Illustration of pitch and roll angle as well as rotation rates around all axes for an azimuth step input command (linear simulation)
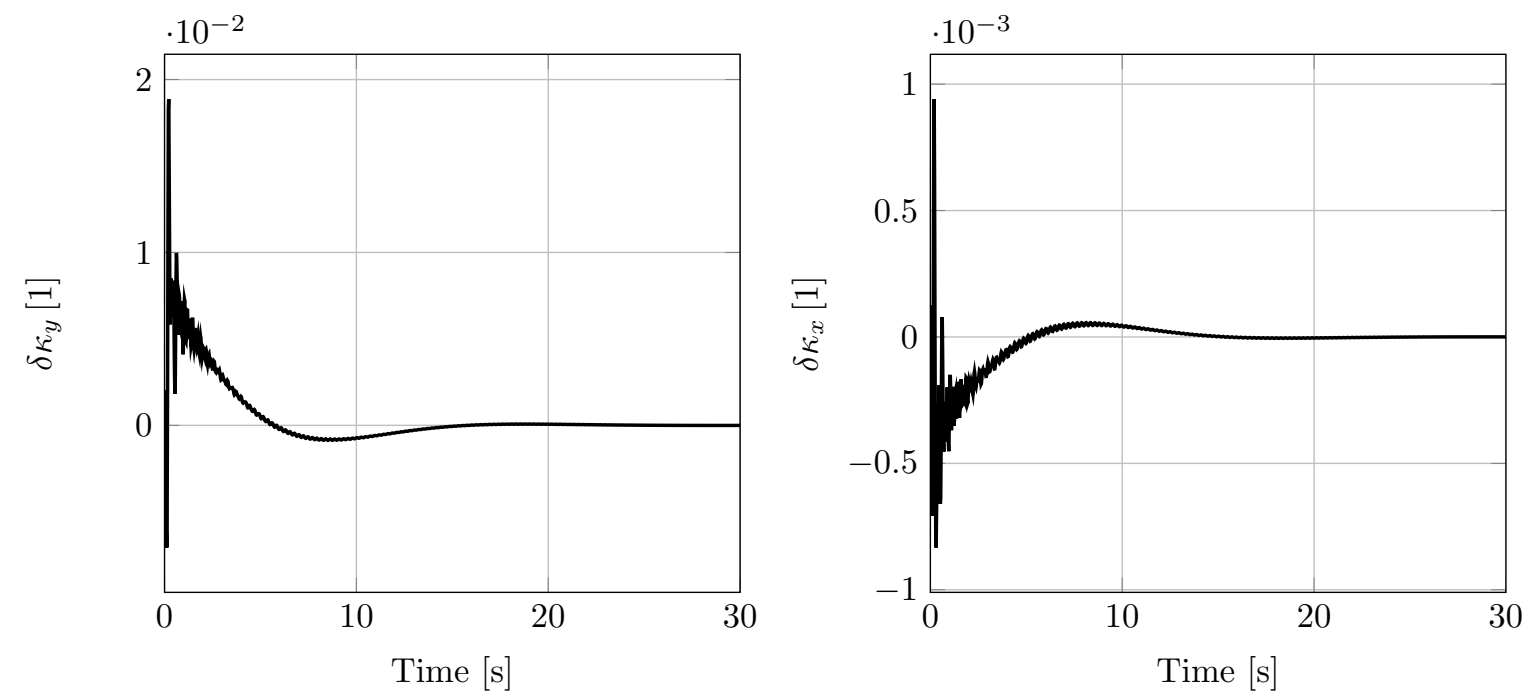

Figure 14: Illustration of curvature at the right wing tip for an azimuth step input command (linear simulation) 
but after a short time this effect is negligible. In summary, the azimuth command controller possesses a good performance and all requirements are fulfilled.

\section{Nonlinear closed loop simulation}

Similar to the linear simulation, the controller are included as state space models in the nonlinear simulation environment UM/NAST of the University of Michigan that was developed in $\mathrm{C}++$. The computation of the control laws was carried out with a fourth-order Runge-Kutta integration routine. The sample rate of the simulation was $100 \mathrm{~Hz}$. Limiters for the maximum pitch and bank angle command are implemented. Figure 15 illustrates the control surface activity and the flight mechanical parameters altitude, azimuth, velocity and sideslip angle are illustrated for a $50 \mathrm{~m}$ step command in altitude from $30 \mathrm{~m}$ to $80 \mathrm{~m}$. The altitude tracking performance is similar to the linear simulation. The deviations in the azimuth angle and velocity are equivalent to the linear simulation and in an acceptable range. This emphasises again the good performance of the speed controller. The behaviour of the sideslip angle is equal in both simulations. Figure 16 shows the pitch and bank angle as well as the rotational rates. The influence of the structural motion to the rigid body motion is faster decaying away than in the linear simulation. In the linear simulation the influence is significant for more than five seconds. In the nonlinear simulation the high oscillation is negligible after one second. The maximum pitch angle is a little bit higher than $10^{\circ}$. This is mainly due to the fact that the derivation from the trimmed conditions is limited and not the total pitch angle.

The results for the azimuth control law are illustrated in Fig. 17 and 18. The time to reach the commanded azimuth angle shorter in the nonlinear simulation than in the linear simulation, but a very high overshoot occurs. The damping of the azimuth motion is very low. Altitude and speed hold is achieved with good performance during a step command in the azimuth angle. Also the sideslip angle is very low that shows good performance of the sideslip angle controller. In Fig. 18 it can be seen that the bank angle is an acceptable range. Due to the low damping of the azimuth motion, oscillations in sideslip angle and bank angle are obviously. In comparison to the linear simulation, the coupling of high frequency oscillations with flight mechanical states is much smaller in the non-linear simulation. Like the altitude controller, the control surface activity is very low for the azimuth control loop.

\section{Conclusion}

In this paper a new approach for the control of a highly flexible aircraft was introduced. A robust innerloop control law based on linear design models was used to achieve stability and reduce the influence of structural motions also for non-modelled nonlinear dynamics. The inner-loop control law was reduced with balanced truncation from 380th to eighth order while robust stability is guaranteed. The outer loops were designed with classical root locus methods for tracking of altitude, azimuth, velocity and sideslip angle. The control laws were analysed and simulated with a linear and a nonlinear simulation model. With regards to nominal and robust stability and control surface deflection, the requirements are fulfilled. All control laws were stable in the nonlinear simulation and aerodynamic surface deflections and thrust commands are very low. Decoupling between structural and rigid body motions was not completely fulfilled relating to the results in the linear simulation. In the non-linear simulation the objectives relating to a decoupling of flight mechanic and structural modes is achieved. In the linear simulation, tracking performance of the outer loops is very satisfying to the requirements. Altitude tracking is also very well achieved in the non-linear simulation. A high overshoot of the yaw angle in nonlinear simulations requires control law modifications. Sideslip and velocity control is satisfactory in linear and nonlinear simulation. In summary the introduced approach for a flight control system of highly flexible aircraft is successful. Tracking performance is achieved. The introduced approach can deal with nonlinear behaviour and a low-order control law that can be realized in practical applications.

\section{References}

\footnotetext{
${ }^{1}$ Brockhaus, R., Alles, W., Luckner, R., Flugregelung, Vol. 3, Springer, 2013.

${ }^{2}$ Cesnik, C. E., et al., X-HALE: A very flexible unmanned aerial vehicle for nonlinear aeroelastic tests, AIAA Journal 50.12: 2820-283, 2012.

${ }^{3}$ Cook, R. G., Palacios, R., and Goulart, P., Robust gust alleviation and stabilization of very flexible aircraft, AIAA Journal 51.1: 330-340, 2013.
} 

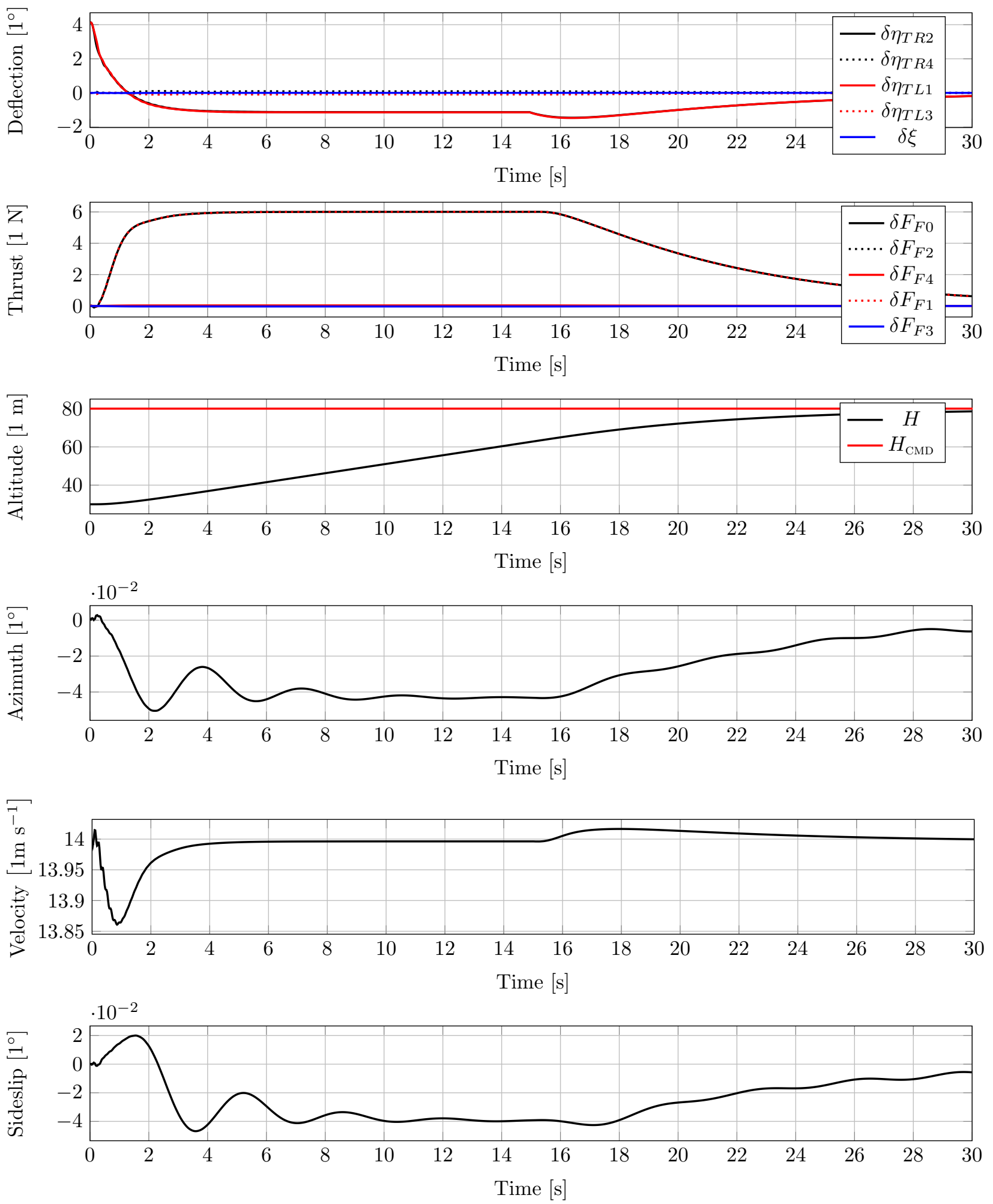

Figure 15: Illustration of control activity and flight mechanical parameter $H, \Psi, V, \beta$ for an altitude step input (non-linear simulation) 

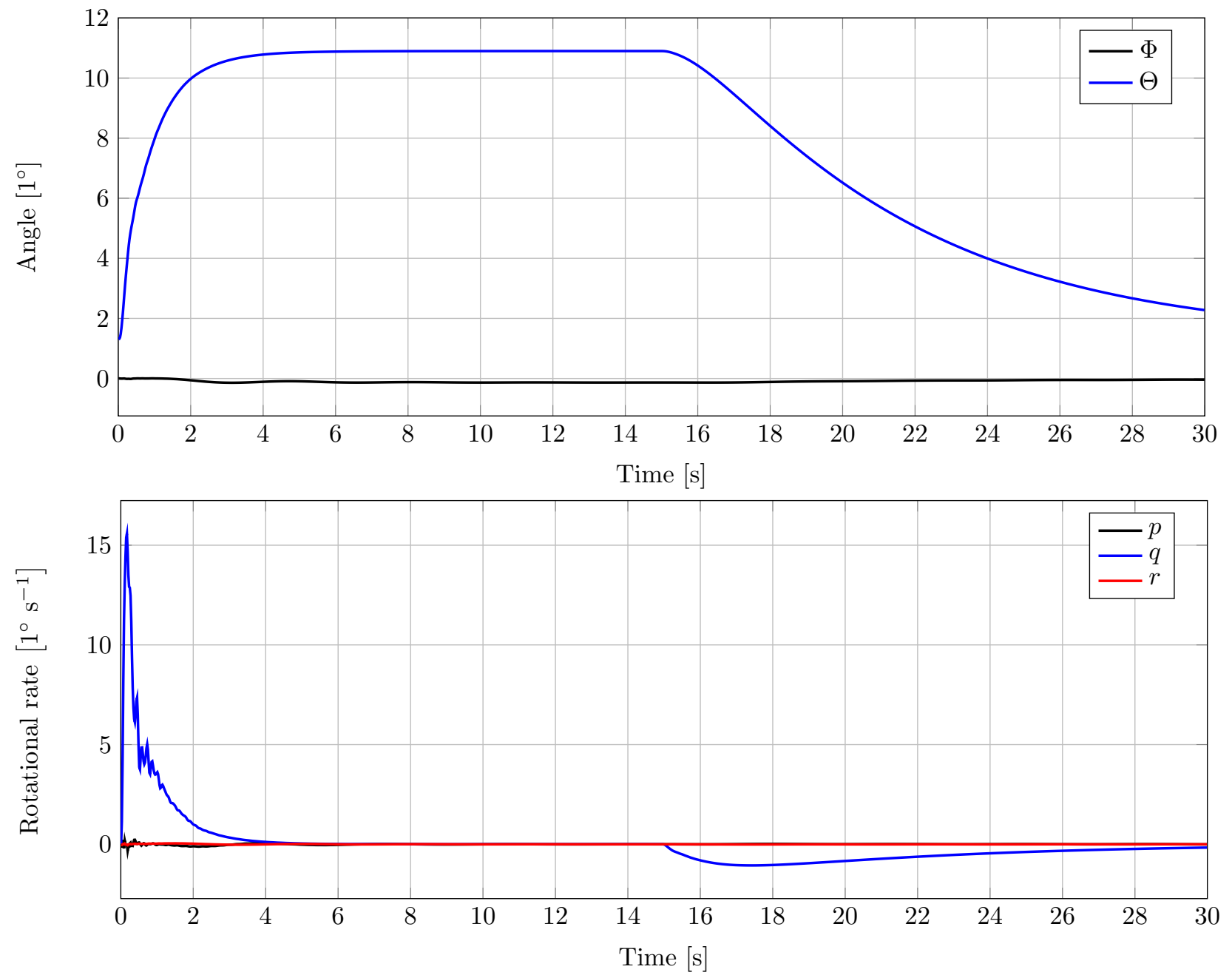

Figure 16: Illustration of pitch and roll angle as well as rotation rates around all axes for an altitude step input command in the nonlinear simulation 

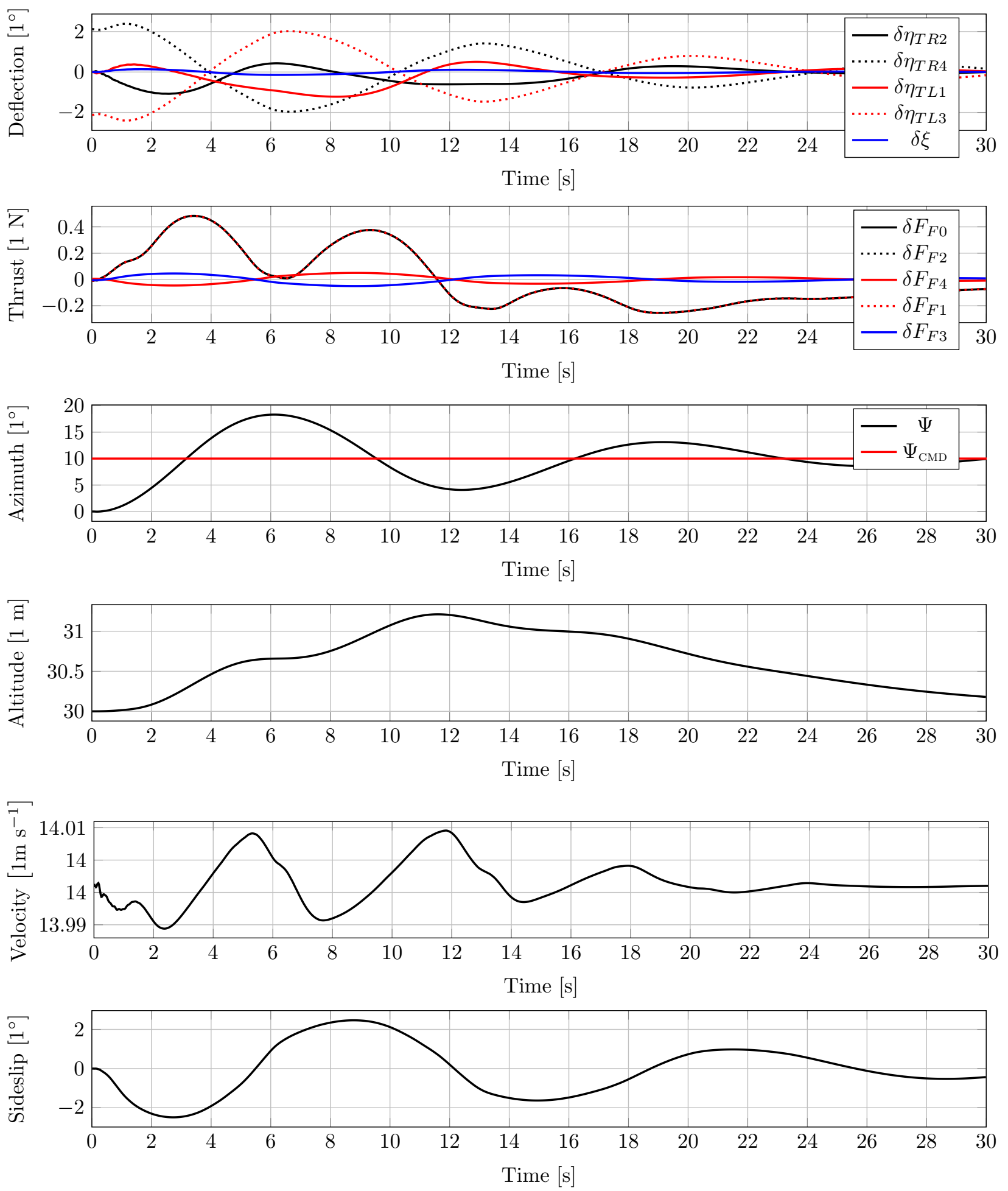

Figure 17: Illustration of control activity and flight mechanical parameter $\Psi, H, V, \beta$ for an azimuth step input command (non-linear simulation) 

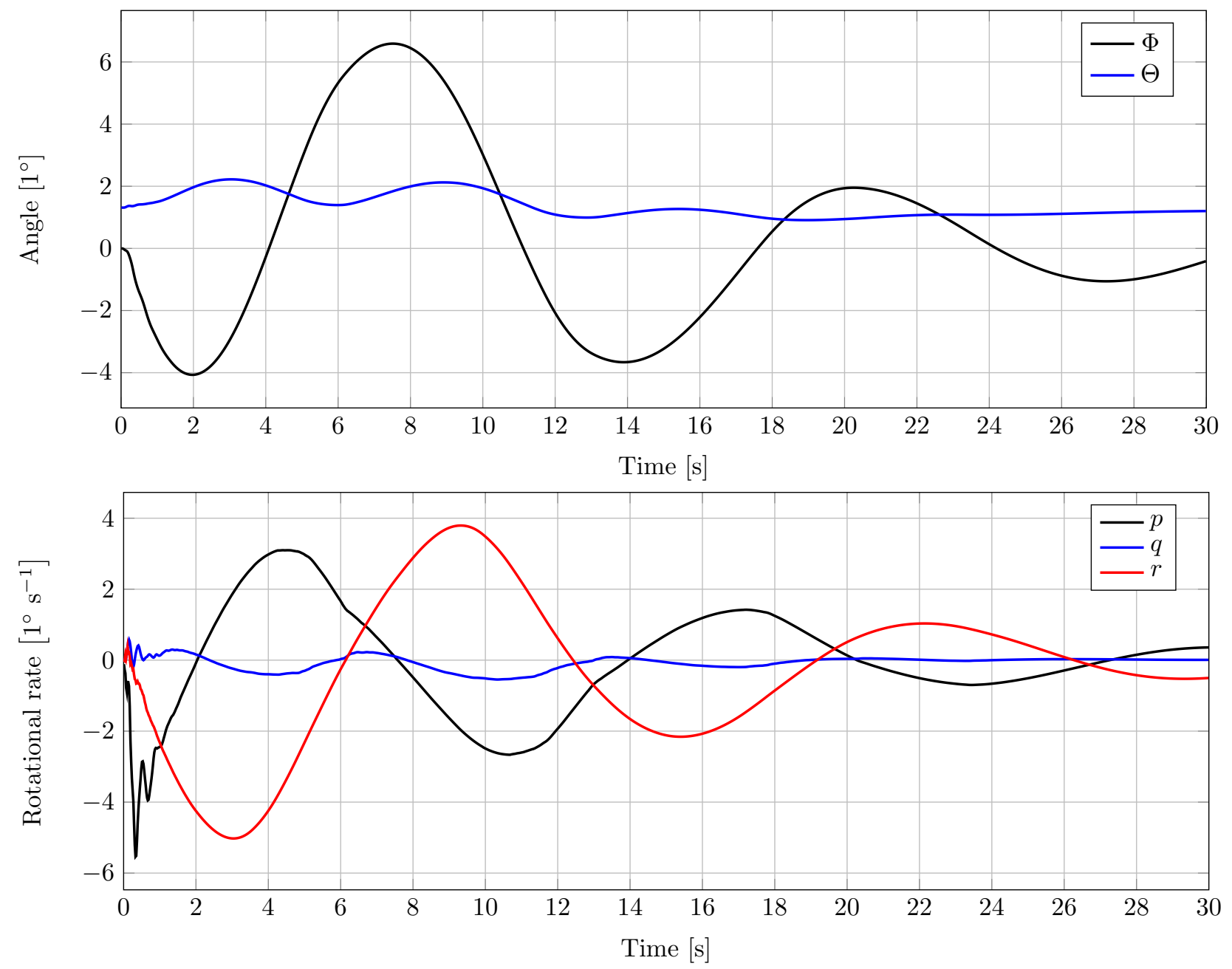

Figure 18: Illustration of pitch and roll angle as well as rotation rates around all axes for an azimuth step input command (non-linear simulation) 
${ }^{4}$ Davey, P., Zephyr HALE UAS, AIAA Infotech@ Aerospace 2007 Conference and Exhibit. 2007.

${ }^{5}$ Dillsaver, M., Cesnik, C. E., and Kolmanovsky, I., Trajectory Control of Very Flexible Aircraft with Gust Disturbance, AIAA Atmospheric Flight Mechanics (AFM) Conference, 2013

${ }^{6}$ Dillsaver, M. J., Gust Response and Control of Very Flexible Aircraft, Ph.D. Dissertation, United States Air Force, 2013.

${ }^{7}$ Gibson, T. E., Annaswamy, A. M., and Lavretsky, E., Modeling for control of very flexible aircraft, AIAA Guidance, Navigation, and Control Conference, 2011.

${ }^{8} \mathrm{Gu}$, D. W., Petkov, P. H., and Konstantinov, M. M., Robust control design with MATLAB®, Springer Science \& Business Media, 2005.

${ }^{9}$ Köthe, A., and Luckner, R., Flight Mechanical Modeling and Analysis of Multi-Body Aircraft, International Forum on Aeroelasticity and Structural Dynamics (IFASD), St. Petersburg, Russia 2015

${ }^{10}$ Pratt, R., Flight control systems: practical issues in design and implementation, IEE, 2000.

${ }^{11}$ Patil, M., and Hodges, D., Flight dynamics of highly flexible flying wings, Journal of Aircraft 43.6 (2006): 1790-1799.

${ }^{12}$ Raghavan, B., and Patil, M. J., Flight control for flexible, high-aspect-ratio flying wings, Journal of guidance, control, and dynamics 33.1: 64-74, 2010.

${ }^{13}$ Shearer, C., and Cesnik, C., Nonlinear flight dynamics of very flexible aircraft, Journal of Aircraft 44.5 (2007): 1528-1545.

${ }^{14}$ Skogestad, S., and Postlethwaite, I., Multivariable feedback control: analysis and design, Vol. 2, Wiley, 2007

${ }^{15}$ Stein, G., Bode lecture: respect the unstable, Proceedings of Conference on Decision and Control, 1989

${ }^{16} \mathrm{Su}, \mathrm{W}$. , and Cesnik, C., Dynamic Response of Highly Flexible Flying Wings, AIAA Journal Vol. 49, No. 2, February 2011

${ }^{17} \mathrm{Su}, \mathrm{W}$., and Cesnik, C., Strain-based geometrically nonlinear beam formulation for modeling very flexible aircraft, International Journal of Solids and Structures 48, 23492360, 2011

${ }^{18}$ Stevens, B. L., and Lewis, F. L., Aircraft control and simulation, Wiley, 2013.

${ }^{19}$ USAF, MIL-F-9490D, Military Specification: Flight Control SystemsGeneral Specifications for Design, Installation, and Test of Piloted Aircraft, 1975. 